\title{
VECTOR BUNDLES, DUALITIES, AND CLASSICAL GEOMETRY ON A CURVE OF GENUS TWO
}

\author{
NGUYỄN QUANG MINH
}

\begin{abstract}
Let $C$ be a curve of genus two. We denote by $\mathcal{S U}_{C}(3)$ the moduli space of semistable vector bundles of rank 3 and trivial determinant over $C$, and by $J^{d}$ the variety of line bundles of degree $d$ on $C$. In particular, $J^{1}$ has a canonical theta divisor $\Theta$. The space $\mathcal{S U}_{C}(3)$ is a double cover of $\mathbb{P}^{8}=|3 \Theta|$ branched along a sextic hypersurface, the Coble sextic. In the dual $\check{\mathbb{P}}^{8}=|3 \Theta|^{*}$, where $J^{1}$ is embedded, there is a unique cubic hypersurface singular along $J^{1}$, the Coble cubic. We prove that these two hypersurfaces are dual, inducing a non-abelian Torelli result. Moreover, by looking at some special linear sections of these hypersurfaces, we can observe and reinterpret some classical results of algebraic geometry in a context of vector bundles: the duality of the Segre-Igusa quartic with the Segre cubic, the symmetric configuration of 15 lines and 15 points, the Weddle quartic surface and the Kummer surface.
\end{abstract}

\section{INTRODUCTION}

The moduli space of vector bundles of rank 2 on a smooth projective curve $C$ has been much studied since its construction in the sixties. it has beautiful connections to the geometry of the $2 \Theta$ linear system. Since the Kummer variety is contained in the above moduli space (with fixed determinant of degree 0), much of the classical Kummer geometry has vector bundle theoretical interpretations. Surprisingly, a similar study of the $3 \Theta$ linear system also reveals connections to classical complex algebraic geometry.

Let $C$ be a smooth complex projective curve of genus 2 and $J^{1}$ the space of divisors of degree 1 on $C$. The variety $J^{1}$ has a canonical Riemann theta divisor $\Theta$. We denote by $\mathcal{S U}_{C}(3)$ the moduli space of semi-stable rank-3 vector bundles on $C$ with trivial determinant. This projective variety of dimension 8 is the double cover of $|3 \Theta| \cong \mathbb{P}^{8}$ branched along a hypersurface of degree 6 that we call the Coble sextic and denote by $\mathcal{C}_{6}$. Moreover $J^{1}$ embeds naturally into $|3 \Theta|^{*} \cong \check{\mathbb{P}}^{8}$, and there is a unique cubic hypersurface $\mathcal{C}_{3}$ in $\check{\mathbb{P}}^{8}$ singular along the embedded $J^{1}$. We call it the Coble cubic. The main result of this paper is the "global" duality:

Theorem 3.4.1, The Coble hypersurfaces $\mathcal{C}_{3}$ and $\mathcal{C}_{6}$ are dual.

This was first conjectured by Dolgachev and mentioned by Laszlo in [Las96]. It was finally proved by Ortega Ortega in her thesis [0005], with the use of some computer calculations. We give here a different "computer-free" proof. The duality then allows us to deduce a non-abelian Torelli result.

Corollary 3.4.4. Let $C$ and $C^{\prime}$ be two smooth projective curves of genus 2. If $\mathcal{S U}_{C}(3)$ is isomorphic to $\mathcal{S U}_{C^{\prime}}(3)$, then $C$ is isomorphic to $C^{\prime}$.

Using the duality and a geometric study of $\mathcal{S U}_{C}(3)$, we can recover a number of classical results related to curves of genus 2 , but in the context of vector bundles.

2000 Mathematics Subject Classification. 14H60, 14J70, 14E20, 14E30, 14C34. 


\section{ACKNOWLEDGEMENTS}

I would like to express my sincere thanks to Igor Dolgachev for all the support, guidance and encouragement during my research. His great insights contributed to my fond interest in the subject.

For the material covered in this treatise, I am greatly indebted to Igor Dolgachev for all the long talks we had, but also to Alessandro Verra, Sławek Rams, Angela Ortega Ortega, Mihnea Popa, Paul Hacking and Ravi Vakil.

\section{PREliminaries: Definitions And Notations}

1.1. The moduli space of vector bundles: generalities. Let $E$ be an algebraic (or holomorphic) vector bundle of rank $r$ on a smooth projective curve $C$ of genus $g(C)=g$. We define its slope of $E$ to be the number

$$
\mu(E)=\frac{\operatorname{deg}(E)}{r}=\frac{\operatorname{deg}(\operatorname{det}(E))}{r} .
$$

A vector bundle $E$ is said to be semi-stable (resp. stable) if for any proper subbundle $F$ the following inequality holds

$$
\mu(F) \leq \mu(E) \quad(\text { resp. } \mu(F)<\mu(E)) .
$$

This notion of stability leads to the Geometric Invariant Theoretical construction of moduli spaces of vector bundles, under the following equivalence relation [Ses67]: every semistable vector bundle $E$ admits a Jordan-Hölder filtration

$$
0=E_{0} \subsetneq E_{1} \subsetneq \cdots \subsetneq E_{k-1} \subsetneq E_{k}=E,
$$

such that each successive quotient $E_{i} / E_{i-1}$ is stable of slope equal to $\mu(E)$, for $i=1, \ldots, k$. We call

$$
\operatorname{gr}(E)=\bigoplus_{i=1}^{k} E_{i} / E_{i-1}
$$

the graded bundle associated to $E$. Finally, two semi-stable vector bundles $E$ and $E^{\prime}$ on $C$ are said to be $S$-equivalent if $\operatorname{gr}(E) \cong \operatorname{gr}\left(E^{\prime}\right)$. We write $E \sim_{S} E^{\prime}$. In particular, two stable bundles $E$ and $E^{\prime}$ are $S$-equivalent if and only if they are isomorphic.

So we denote by $\mathcal{U}_{C}(r, d)$ the moduli of $S$-equivalence classes of semi-stable vector bundles on $C$ of rank $r$ and degree $d$. If we fix a line bundle $L$ on the curve $C$, we denote by $\mathcal{S U}_{C}(r, L)$ the moduli space of $S$-equivalence classes of semi-stable vector bundles on $C$ of rank $r$ and fixed determinant $L$. The singular locus of these moduli spaces corresponds exactly to decomposable bundles, i.e. strictly semi-stable bundles, except when $g(C)=r=2$ and $d$ is even.

Tensoring by a line bundle $M$ on $C$ induces an isomorphism

$$
\mathcal{S U}_{C}(r, L) \stackrel{\sim}{\rightarrow} \mathcal{S U}_{C}\left(r, L \otimes M^{r}\right),
$$

so when we do not want to specify $L$, we will just write $\mathcal{S U}_{C}(r, d)$, where $d=\operatorname{deg}(L)$.

In [DN89], Drezet and Narasimhan prove that these spaces are locally factorial and describe the Picard groups. For the moduli space with trivial determinant $\mathcal{S U}_{C}(r)=$ $\mathcal{S U}_{C}\left(r, \mathcal{O}_{C}\right)$, if we fix a general line bundle $L$ of degree $g-1$, then the set

$$
\Delta_{L}=\left\{E \in \mathcal{S U}_{C}(r): h^{0}(C, E \otimes L)>0\right\}
$$

is a divisor on $\mathcal{S U}_{C}(r)$ whose isomorphism class does not depend on $L$. We write

$$
\Theta^{\text {gen }}=\mathcal{O}_{\mathcal{S U}_{C}(r)}\left(\Delta_{L}\right)
$$


for the corresponding line bundle (isomorphism class). Then the Picard group of $\mathcal{S U}_{C}(r)$ is infinite cyclic and generated by $\Theta^{\text {gen }}$.

This ample generator is called the generalized theta divisor (or determinant bundle) for it generalizes the traditional notion of theta divisor on Jacobians of curves. Indeed the variety $J^{g-1}$, seen as a space of line bundles of degree $g-1$ on $C$, has a canonical Riemann theta divisor $\Theta$ defined as

$$
\Theta=\left\{L \in J^{g-1}: h^{0}(C, L)>0\right\} .
$$

For any $E \in \mathcal{S U}_{C}(r)$, we define

$$
D_{E}=\left\{L \in J^{g-1}: h^{0}(C, E \otimes L)>0\right\} \subset J^{g-1} .
$$

It is known that $D_{E}$ is either the whole space $J^{g-1}$ or a divisor of the linear system $|r \Theta|$. The former case only happens for special $E \in \mathcal{S U}_{C}(r)$, so we get a rational map $\Phi_{r}$ :

$$
\begin{aligned}
\Phi_{r}: \mathcal{S U}_{C}(r) & \rightarrow|r \Theta|, \\
E & \longmapsto D_{E}=\left\{L \in J^{g-1}: h^{0}(C, E \otimes L)>0\right\} .
\end{aligned}
$$

This map is a canonical description of the map defined by the linear system $\left|\Theta^{\text {gen }}\right|$. This follows from a theorem of Beauville, Narasimhan and Ramanan [BNR89] which states that there is a canonical isomorphism

$$
H^{0}\left(\mathcal{S U}_{C}(r), \Theta^{\text {gen }}\right)^{*} \cong H^{0}\left(J^{g-1}(C), r \Theta\right) .
$$

Example 1.1.1. For a curve $C$ of genus 2, the rational map $\Phi_{2}: \mathcal{S U}_{C}(2) \rightarrow|2 \Theta|$ is an isomorphism (see [NR69]), i.e. $\mathcal{S U}_{C}(2) \cong \mathbb{P}^{3}$.

1.2. Origins and motivations: The Coble quartic. Before we start discussing vector bundles of rank 3, we look at what is already known in rank 2 and the main motivating result.

A concrete geometric description of moduli spaces of vector bundles with fixed determinant on a given curve $C$ is known in the following cases:

- $g(C)=0 . \mathcal{S U}_{C}(r, d)$ is either empty or just a point (when $r \mid d$ ).

- $g(C)=1$. $\mathcal{S U}_{C}(r, d) \cong \mathbb{P}^{h-1}$, where $h=(r, d)$ [Ati57, Tu93].

- $g(C)=2, r=2$. $\mathcal{S U}_{C}(2) \cong \mathbb{P}^{3}$ and $\mathcal{S U}_{C}(2,1)$ is isomorphic to the intersection of 2 quadrics in $\mathbb{P}^{5}$ [NR69, New68].

Then let $C$ be non-hyperelliptic of genus 3 and $r=2$. The divisor $2 \Theta$ on the Jacobian $J^{2}$ defines a map from $J^{2}$ to $|2 \Theta|^{*}$ whose image is the Kummer variety $\mathcal{K}_{2}$. In [Cob61], Coble shows that there is a unique quartic hypersurface-now called the Coble quarticin $|2 \Theta|^{*} \cong \mathbb{P}^{7}$ singular exactly along $\mathcal{K}_{2}$. On the other hand, Narasimhan and Ramanan [NR87] study the natural map

$$
\Phi_{2}: \mathcal{S U}_{C}(2) \rightarrow|2 \Theta|
$$

and prove that it embeds $\mathcal{S U}_{C}(2)$ as a quartic hypersurface of $|2 \Theta|$ singular exactly along the Kummer variety. So by uniqueness, it follows that $\mathcal{S U}_{C}(2)$ is isomorphic to the Coble quartic. Using the moduli interpretation (and the Wirtinger duality), Pauly proves that the Coble quartic is self-dual [Pau02].

The beautiful connections found in the geometry of the Coble quartic provide the inspiration for the present account. 
1.3. The Coble sextic and the Coble cubic. Let $C$ be a smooth projective curve of genus 2 , therefore hyperelliptic. We wish to study the moduli space $\mathcal{S U}_{C}(3)$ of rank-3 vector bundles on $C$ with trivial determinant. As usual, $\Theta$ denotes the canonical Riemann theta divisor of $J^{1}$ and $\Theta^{\text {gen }}$ the generalized theta divisor. The main tool to study $\mathcal{S U}_{C}(3)$ is the map $\Phi_{3}$ of (1).

Theorem 1.3.1. The map $\Phi_{3}: \mathcal{S U}_{C}(3) \rightarrow|3 \Theta| \cong \mathbb{P}^{8}$ is a finite map of degree 2 .

A first unpublished proof was given by Butler and Dolgachev using the Verlinde formula, then Laszlo produced another beautiful proof in [Las96] by making a Hilbert polynomial computation.

Let $\mathcal{I}_{J^{1}}$ be the ideal sheaf corresponding to the tricanonical embedding $J^{1} \hookrightarrow \check{\mathbb{P}}^{8}$. Since $J^{1}$ is projectively normal [Koi76], it follows that

$$
h^{0}\left(\check{\mathbb{P}}^{8}, \mathcal{I}_{J^{1}}(2)\right)=h^{0}\left(\check{\mathbb{P}}^{8}, \mathcal{O}(2)\right)-h^{0}\left(J^{1}, \mathcal{O}_{J^{1}}(6 \Theta)\right)=9 .
$$

Remark 1.3.2. We use the notation $\mathbb{P}^{8}$ for $|3 \Theta|$, and therefore $\check{\mathbb{P}}^{8}$ for for the dual projective space $|3 \Theta|^{*}$.

In [Cob17, Coble produces nine quadrics that cut out the embedded Jacobian $J^{1}$, even scheme-theoretically [Bar95]. More remarkably, it turns out that the nine quadrics are the nine partial derivatives of a cubic polynomial, leading to the conclusion that there is a unique cubic hypersurface in $\check{\mathbb{P}}^{8}$ singular exactly along $J^{1}$ [Bea03].

Definition 1.3.3. The cubic hypersurface singular exactly along $J^{1}$ is called the Coble cubic. We will denote it by $\mathcal{C}_{3}$.

Back to the double cover $\Phi_{3}$, an easy computation using the Hurwitz formula and the fact that the canonical bundle of $\mathcal{S U}_{C}(3)$ is $\left(\Theta^{\text {gen }}\right)^{-6}$ shows that the branch locus of $\Phi_{3}$ is a sextic hypersurface in $|3 \Theta| \cong \mathbb{P}^{8}$.

Definition 1.3.4. The branch divisor of $\Phi_{3}: \mathcal{S U}_{C}(3) \rightarrow|3 \Theta|$ is called the Coble sextic. We will denote by it $\mathcal{C}_{6}$.

The name comes from Dolgachev's conjecture that this branch locus is the dual variety of the Coble cubic, a statement clearly motivated by the Coble quartic (see Section 1.2) and its self-duality.

\section{A First Vector Bundle Interpretation of Classical Geometry}

2.1. Natural group actions on $|3 \Theta|$ and equations of the Coble cubic. We will now describe two natural group actions on $|3 \Theta|$ (and also on $|3 \Theta|^{*}$ ). First, there is the involution of the double cover map $\Phi_{3}$. The standard adjunction involution of $J^{1}, L \mapsto \omega_{C} \otimes L^{-1}$, induces involutions of the vector spaces $H^{0}\left(J^{1}, \mathcal{O}(3 \Theta)\right)$ and $H^{0}\left(J^{1}, \mathcal{O}(3 \Theta)\right)^{*}$, and then of the projectivizations $|3 \Theta| \cong \mathbb{P}^{8}$ and $|3 \Theta|^{*} \cong \check{\mathbb{P}}^{8}$. We denote by $\tau$ both the involutions of $|3 \Theta|$ and $|3 \Theta|^{*}$. Let also $\tau^{\prime}$ be the involution of $\mathcal{S U}_{C}(3)$ given by $E \mapsto \tau^{\prime}(E)=E^{*}$, where $E^{*}$ denotes the dual vector bundle of $E$, and let $h$ be the hyperelliptic involution of $C$. Then, the double cover involution $\sigma$ is (see for instance [0005])

$$
\sigma=\tau^{\prime} \circ h^{*}=h^{*} \circ \tau^{\prime}: E \mapsto h^{*} E^{*},
$$

that is, the ramification locus of $\mathcal{S U}_{C}(3)$ corresponds exactly to

$$
\left\{E \in \mathcal{S U}_{C}(3): \sigma(E)=h^{*} E^{*} \sim_{S} E\right\} \cong \mathcal{C}_{6} .
$$


By Riemann-Roch, we see that $\Phi_{3}$ is $\tau$-equivariant, i.e.

$$
\tau \circ \Phi_{3}=\Phi_{3} \circ \tau^{\prime} .
$$

This implies in particular that if we identify the branch locus and the ramification locus $\mathcal{C}_{6}$ of the double cover $\Phi_{3}$ of $\mathbb{P}^{8}$, then

$$
\operatorname{Fix}(\tau) \cap \mathcal{C}_{6} \cong \operatorname{Fix}\left(\tau^{\prime}\right) \cap \mathcal{C}_{6},
$$

where $\operatorname{Fix}(\tau)\left(\right.$ resp. $\left.\operatorname{Fix}\left(\tau^{\prime}\right)\right)$ denotes the fixed locus of $\tau$ (resp. $\tau^{\prime}$ ). So we can also describe points of $\operatorname{Fix}(\tau)$ or $\mathcal{C}_{6} \subset \mathbb{P}^{8}$ as vector bundles.

Another natural group acting on $|3 \Theta| \cong \mathbb{P}^{8}$ is the group $J_{3}$ of 3 -torsion points of the Jacobian $J$. We know that $J_{3}$ is symplectically isomorphic to the group $\left(\mathbb{F}_{3}\right)^{4}$, where $\mathbb{F}_{3}$ denotes the cyclic group of order 3 . The choice of such an isomorphism is called a level-3 structure and corresponds to the choice of a nice basis for $H^{0}\left(J^{1}, \mathcal{O}(3 \Theta)\right)$ in the following sense (see for instance [LB92]):

Theorem 2.1.1. Let us fix a symplectic isomorphism $\phi: J_{3} \rightarrow\left(\mathbb{F}_{3}\right)^{4}$, where the symplectic structure on $J_{3}$ is the Weil pairing defined by the cup product on $H^{1}\left(C, \mathbb{F}_{3}\right) \cong J_{3}$. Then there exists a unique isomorphism $|3 \Theta| \stackrel{\sim}{\rightarrow} \mathbb{P}^{8}$ which is $\phi$-equivariant with respect to the action of $J_{3}$ on $|3 \Theta|$ and that of $\left(\mathbb{F}_{3}\right)^{4}$ on $\mathbb{P}^{8}$ under the Schrödinger representation.

From the Schrödinger representation of $\left(\mathbb{F}_{3}\right)^{4}$, coordinates on $\mathbb{C}^{9}$ can be written $X_{b}$, for $b \in\left(\mathbb{F}_{3}\right)^{2}$. With these coordinates and using the quadrics derived in [Cob17], the Coble cubic $\mathcal{C}_{3}$ is defined by the polynomial

$$
\begin{aligned}
\frac{\alpha_{0}}{3} \sum_{b \in\left(\mathbb{F}_{3}\right)^{2}} X_{b}^{3} & +2 \alpha_{1}\left(X_{00} X_{01} X_{02}+X_{10} X_{11} X_{12}+X_{20} X_{21} X_{22}\right) \\
& +2 \alpha_{2}\left(X_{00} X_{10} X_{20}+X_{01} X_{11} X_{21}+X_{02} X_{12} X_{22}\right) \\
& +2 \alpha_{3}\left(X_{00} X_{11} X_{22}+X_{01} X_{12} X_{20}+X_{10} X_{21} X_{02}\right) \\
& +2 \alpha_{4}\left(X_{00} X_{12} X_{21}+X_{01} X_{10} X_{22}+X_{02} X_{11} X_{20}\right)
\end{aligned}
$$

where $\alpha_{0}, \ldots, \alpha_{4}$ are parameters for the genus- 2 curve $C$. Moreover, $J_{3}$ acts by tensor product on $\mathcal{S U}_{C}(3)$ and it is easy to prove the following from (1) and (5).

Proposition 2.1.2. The double cover map $\Phi_{3}$ is $J_{3}$-equivariant. In addition, the Coble sextic $\mathcal{C}_{6}$ and the Coble cubic $\mathcal{C}_{3}$ are both $J_{3}$-invariant.

2.2. The Igusa-Segre quartic. The fixed locus of the involution $\tau$ of $|3 \Theta|$ is a disjoint union of projective spaces:

$$
\operatorname{Fix}(\tau)=\operatorname{Fix}(\tau)_{+} \sqcup \operatorname{Fix}(\tau)_{-}, \quad \text { i.e } \operatorname{Fix}(\tau)=\mathbb{P}_{+}^{4} \sqcup \mathbb{P}_{-}^{3} .
$$

In this section, we state some results about the geometry of the intersections $\mathcal{V}=\mathcal{C}_{6} \cap \mathbb{P}_{+}^{4}$ and $\mathfrak{H}=\mathcal{C}_{6} \cap \mathbb{P}_{-}^{3}$. Notice first that the two fixed component $\mathbb{P}_{+}^{4}$ and $\mathbb{P}_{-}^{3}$ are not contained in $\mathcal{C}_{6}$ ([INgu05, Proposition III.1] and an easy consequence of its proof).

Let us fix a level-2 structure on $J$ or rather $J^{1}$. This is a symplectic isomorphism

$$
\xi: J_{2} \rightarrow\left(\mathbb{F}_{2}\right)^{4},
$$

where $J_{2}$ denotes the group of 2-torsion points of $J$. Moreover, $J_{2}$ acts on the moduli space $\mathcal{S U}_{C}(2)$ of rank-2 bundles on $C$ by tensor product. As in Theorem 2.1.1 and Example 1.1.1, the level-2 structure $\xi$ determines an equivariant isomorphism $\mathcal{S U}_{C}(2) \stackrel{\sim}{\rightarrow} \mathbb{P}^{3}$ that we embed into $\mathcal{V}$ by defining

$$
V_{0}=\left\{\mathcal{O}_{C} \oplus F: F \in \mathcal{S U}_{C}(2)\right\} \cong \mathbb{P}^{3} \subset \mathcal{V}
$$


Another way to produce vector bundles in $\mathcal{V}$ is to take symmetric powers of vector bundles of $\mathcal{S U}_{C}(2)$. The image of the map

$$
\operatorname{Sym}^{2}: \mathcal{S U}_{C}(2) \rightarrow \mathcal{S U}_{C}(3), F \mapsto \operatorname{Sym}^{2} F
$$

turns out to be to a well-known quartic hypersurface of $\mathbb{P}^{4}$, the Igusa-Segre quartic. This quartic, denoted $\mathcal{I}_{4}$, has an interpretation as the Satake compactification of the moduli space $\mathcal{A}_{2}^{*}(2)$ of abelian surfaces with level-2 structure [Gee82].

Theorem 2.2.1 ([NR03]). The scheme $\mathcal{V}$, of degree 6 in $\mathbb{P}_{+}^{4}$, is the union of the Igusa-Segre quartic $\mathcal{I}_{4}$ and the double hyperplane $V_{0}$. Moreover, the hyperplane $V_{0}$ is tangent to $\mathcal{I}_{4}$ at the point corresponding to the trivial vector bundle $\mathcal{O}_{C}^{\oplus 3}$ from the $\mathcal{S U}_{C}(3)$ perspective, but also to the point $(J, \xi)$ in the moduli space $\mathcal{I}_{4}=\mathcal{A}_{2}^{*}(2)$.

The geometry of $\mathcal{I}_{4}$ is beautiful and well known. Its singular locus consists of 15 lines meeting in 15 points (or nodes), all fitting in a symmetric $\left(15_{3}\right)$-configuration: on each line there are exactly 3 nodes and through each node pass exactly 3 lines. However, we can identify the 15 lines (and 15 nodes) in terms of vector bundles. For each $\epsilon \in J_{2}-\{0\}$, we set

$$
V_{\epsilon}=\left\{L_{\epsilon} \oplus F: F \in \mathcal{S U}_{C}(2, \epsilon) \text { and } t_{\epsilon}(F)=F\right\} \cong \mathbb{P}^{1} \sqcup \mathbb{P}^{1},
$$

where $t_{\epsilon}$ is the translation morphism of $J^{1}$ acting on $\mathcal{S U}_{C}(2, \epsilon)$ by tensor product.

So $V_{\epsilon}$ is the disjoint union of two lines: one is in $\mathbb{P}_{+}^{4}$ and the other in $\mathbb{P}_{-}^{3}$. Moreover, the 15 lines lying in $\mathbb{P}_{+}^{4}$ are exactly the 15 lines of the singular locus of the Igusa-Segre quartic $\mathcal{I}_{4}$. This in turn allows us to understand the other intersection.

Theorem 2.2.2 ([NR03]). The surface $\mathfrak{H}=\mathcal{C}_{6} \cap \mathbb{P}_{-}^{3}$ is a general hexahedron, i.e. the union of 6 planes in $\mathbb{P}_{-}^{3}$ in general position.

Interestingly, intersecting the Coble sextic with $\mathbb{P}_{+}^{4}$ or $\mathbb{P}_{-}^{3}$ enables us to recover the original curve $C$. In $\mathbb{P}_{+}^{4}$ indeed, there is a natural Kummer surface

$$
\mathcal{K}^{\prime}=\left\{\mathcal{O}_{C} \oplus L \oplus L^{-1}: L \in J\right\}=\mathcal{I}_{4} \cap V_{0},
$$

which together with the tangency point-corresponding to $(J, \xi)$ from the moduli interpretation of $\mathcal{I}_{4}$-completely determines $C$. Retrieving $C$ from $\mathbb{P}_{-}^{3}$ will follow from Theorem 4.2.1.

\section{The Duality of the Coble Hypersurfaces}

The main result of this section is Theorem 3.4.1. Our proof will not make use of computer calculations, unlike A. Ortega Ortega's [0005].

3.1. The degree of the singular locus $\Sigma$. We first analyze the singular locus $\Sigma=\operatorname{Sing}\left(\mathcal{C}_{6}\right)$ of the Coble sextic. Since the target space of the double cover $\Phi_{3}$ is smooth (just $\mathbb{P}^{8}$ ), we know that the singular locus $\Sigma$ of the branch divisor is exactly the singular locus $\Sigma^{\prime}$ of the covering space $\mathcal{S U}_{C}(3)$ corresponding to strictly semi-stable vector bundles. We will keep the two notations, $\Sigma$ and $\Sigma^{\prime}$, in order to make clear in what space we are.

Lemma 3.1.1. The determinant map

$$
\text { det : } \mathcal{U}_{C}(2,0) \rightarrow J, F \mapsto \operatorname{det}(F)
$$

is a $\mathbb{P}^{3}$-fibration: for $a \in J$, the fiber over a is $\mathcal{S U}_{C}(2, a) \cong \mathbb{P}^{3}$. In particular, $\mathcal{U}_{C}(2,0)$ is a smooth variety of dimension 5 . 
Proof. The map

$$
\begin{aligned}
\psi: \mathcal{S U}_{C}(2) \times J & \stackrel{16: 1}{\longrightarrow} \mathcal{U}_{C}(2,0) \\
(F, L) & \longmapsto F \otimes L
\end{aligned}
$$

is an étale covering. Indeed, if $F \otimes L \cong F^{\prime} \otimes L^{\prime}$, then we take the determinants and get $L^{\prime} \otimes L^{-1}=\epsilon \in J_{2}$. So $L^{\prime}=L \otimes \epsilon$ and $F^{\prime}=F \otimes \epsilon$. Therefore $\mathcal{U}_{C}(2,0)$ is the quotient of the trivial projective bundle $\mathcal{S U}_{C}(2) \times J$ under the proper and discontinuous diagonal action of $J_{2}$. Thus the conclusion follows.

Since $\mathcal{U}_{C}(2,0)$ is smooth, it follows the map

$$
\nu: \mathcal{U}_{C}(2,0) \rightarrow \Sigma^{\prime}, F \mapsto F \oplus \operatorname{det}(F)^{*}
$$

is a resolution of singularities of $\Sigma^{\prime}$, as it is clearly surjective and injective on the open locus of stable bundles of $\mathcal{U}_{C}(2,0)$. Therefore via $\nu$ we get:

$$
\operatorname{deg}(\Sigma)=\operatorname{deg}_{\mathbb{P}^{8}}\left(\Sigma \cdot H^{5}\right)=\operatorname{deg}_{\mathcal{U}_{C}(2,0)}\left(\nu^{*}\left(\Theta^{\text {gen }}\right)^{5}\right)
$$

where $H$ is the class of a hyperplane in $\mathbb{P}^{8}$. Indeed, since $\Sigma=\Phi_{3 *} \Sigma^{\prime}$, we apply the projection formula and use the resolution map $\nu$ to see that

$$
\Sigma \cdot\left(H^{5}\right)=\Sigma^{\prime} \cdot\left(\Phi_{3}^{*} H\right)^{5}=\Sigma^{\prime} \cdot\left(\Theta^{\text {gen }}\right)^{5}=\nu_{*} U_{C}(2,0) \cdot\left(\Theta^{\text {gen }}\right)^{5} .
$$

But for a fixed line bundle $L \in J^{1}, \Delta_{L}=\left\{E \in \mathcal{S U}_{C}(3): H^{0}(C, E \otimes L) \neq 0\right\}$ is a divisor representing $\Theta^{\text {gen }}$, so

$$
\begin{aligned}
& \nu^{*}\left(\Delta_{L}\right)=\left\{F \in \mathcal{U}_{C}(2,0): H^{0}\left(C,(F \otimes L) \oplus\left(\operatorname{det}(F)^{*} \otimes L\right)\right) \neq 0\right\}, \\
&=\left\{F \in \mathcal{U}_{C}(2,0): H^{0}(C, F \otimes L) \neq 0\right\} \\
&\left.\cup\left\{F \in \mathcal{U}_{C}(2,0): H^{0}\left(C, \operatorname{det}(F)^{*} \otimes L\right) \neq 0\right)\right\} .
\end{aligned}
$$

To deal with this, we define the map $\pi$ as the following composition:

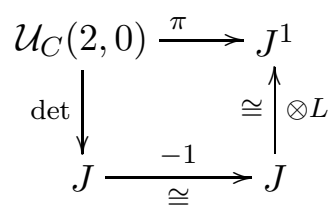

so that $\pi$ is also a $\mathbb{P}^{3}$-fibration. The moduli space $\mathcal{U}_{C}(2,0)$ has a generalized theta divisor $\Theta_{\mathcal{U}}^{\mathrm{gen}}$, associated to the divisor

$$
\Delta_{L}^{\prime}=\left\{F \in U_{C}(2,0): H^{0}(C, F \otimes L) \neq 0\right\} \subset \mathcal{U}_{C}(2,0) .
$$

So we see that at the divisorial level (or set-theoretically) $\nu^{*}\left(\Delta_{L}\right)=\Delta_{L}^{\prime} \cup \pi^{*}(\Theta)$, and as an isomorphism class of line bundles on $\mathcal{U}_{C}(2,0)$,

$$
\nu^{*}\left(\Theta^{\text {gen }}\right)=\Theta_{\mathcal{U}}^{\text {gen }}+\pi^{*}(\Theta) .
$$

Proposition 3.1.2. The degree of the singular locus $\Sigma$ of $\mathcal{C}_{6}$ in $\mathbb{P}^{8}$ is

$$
\operatorname{deg}(\Sigma)=45 .
$$


Proof. Putting (12) and (10) together, we obtain

$$
\begin{aligned}
\operatorname{deg}(\Sigma)= & \operatorname{deg}_{\mathcal{U}_{C}(2,0)}\left(\left[\nu^{*}\left(\Theta^{\text {gen }}\right)\right]^{5}\right)=\operatorname{deg}_{\mathcal{U}_{C}(2,0)}\left(\left[\Theta_{\mathcal{U}}^{\text {gen }}\right]+\left[\pi^{*}(\Theta)\right]\right)^{5} \\
= & \sum_{i=0}^{5}\left(\begin{array}{c}
5 \\
i
\end{array}\right) \operatorname{deg}_{\mathcal{U}_{C}(2,0)}\left(\left[\Theta_{\mathcal{U}}^{\text {gen }}\right]^{i} \cdot\left[\pi^{*}(\Theta)\right]^{5-i}\right) \\
= & \operatorname{deg}_{\mathcal{U}_{C}(2,0)}\left(\left[\Theta_{\mathcal{U}}^{\text {gen }}\right]^{5}\right)+5 \operatorname{deg}_{\mathcal{U}_{C}(2,0)}\left(\left[\Theta_{\mathcal{U}}^{\text {gen }}\right]^{4} \cdot\left[\pi^{*}(\Theta)\right]\right) \\
& +10 \operatorname{deg}_{\mathcal{U}_{C}(2,0)}\left(\left[\Theta_{\mathcal{U}}^{\text {gen }}\right]^{3} \cdot\left[\pi^{*}(\Theta)\right]^{2}\right) .
\end{aligned}
$$

There are three terms in the sum. The technical albeit relatively easy computations can be found in [Ngu05, Lemmas V.6, V.7, V.8] and make use of Lemma 3.1.1 and the étale covering $\psi$ of (8). We hence gather the terms to find that

$$
\operatorname{deg}(\Sigma)=1 \times 5+5 \times 4+10 \times 2=45 .
$$

3.2. A map given by quadrics. Let $G$ be the homogeneous cubic polynomial defining the Coble cubic $\mathcal{C}_{3}$. The motivation here is to interpret in terms of vector bundles the dual map

$$
\begin{aligned}
\mathcal{D}: \mathcal{C}_{3} \subset|3 \Theta|^{*} & \rightarrow|3 \Theta| \\
p & \longmapsto T_{p}\left(\mathcal{C}_{3}\right)=\left[\frac{\partial G}{\partial X_{i}}\right]_{i=0, \ldots 8}
\end{aligned}
$$

given by quadrics. So we are trying to construct a rational map $\Psi: \mathcal{C}_{3} \rightarrow-\rightarrow \mathcal{S U}_{C}(3)$.

We know that $C \cong \Theta \subset J^{1}$. So for every $a \in J$, we write

$$
C_{a}=\Theta+a \subset J^{1} \text { and } \vartheta_{a}=\mathcal{O}_{C_{a}}\left(\left.\Theta\right|_{C_{a}}\right) .
$$

Note that $\vartheta_{a}$ is a line bundle of degree 2 on $C_{a}$ so by Riemann-Roch, we get $h^{0}\left(C_{a}, \vartheta_{a}^{3}\right)=5$ and we denote by $\check{\mathbb{P}}_{a}^{4}=\left|\vartheta_{a}^{3}\right|^{*}$ the linear span of $C_{a}$ in $|3 \Theta|^{*} \cong \check{\mathbb{P}}^{8}$.

Proposition 3.2.1. The span $\check{\mathbb{P}}_{a}^{4}$ of $C_{a}$ in $|3 \Theta|^{*}$ lies in $\mathcal{C}_{3}$.

Proof. Suppose $\check{\mathbb{P}}_{a}^{4} \nsubseteq \mathcal{C}_{3}$, then $\check{\mathbb{P}}_{a}^{4} \cap \mathcal{C}_{3}=V_{3}$ is a cubic threefold of $\check{\mathbb{P}}_{a}^{4}$. Since $\mathcal{C}_{3}$ is singular exactly along $J^{1}$, then $C_{a} \subset \operatorname{Sing}\left(V_{3}\right)$, so a secant line $\ell$ to $C_{a}$ must lie in $V_{3}$. Therefore the secant threefold $\operatorname{Sec}\left(C_{a}\right)$ ( $C_{a}$ does not lie in a plane) is contained in $V_{3}$. We will get a contradiction by showing that $\operatorname{deg} \operatorname{Sec}\left(C_{a}\right)=8$. Indeed, let $\ell$ be a general line in $\check{\mathbb{P}}_{a}^{4}$, it intersects $\operatorname{Sec}\left(C_{a}\right)$ at $d$ points. Therefore, when we project from $\ell, C_{a}$ is mapped to a plane sextic curve of geometric genus two with $d$ nodes. Since the arithmetic genus of a plane sextic curve is 10 , we see that $d=8$.

Let $x \in \check{\mathbb{P}}_{a}^{4}-C_{a}$. It corresponds to a hyperplane $V_{x}$ in $H^{0}\left(C_{a}, \vartheta_{a}^{3}\right)$ :

$$
0 \rightarrow V_{x} \stackrel{j_{x}}{\rightarrow} H^{0}\left(C_{a}, \vartheta_{a}^{3}\right) \stackrel{x}{\rightarrow} \mathbb{C} \rightarrow 0 .
$$

Since $\vartheta_{a}{ }^{3}$ is very ample on $C_{a}$ and $x \notin C_{a}, V_{x}$ generates $\vartheta_{a}{ }^{3}$. We write down the evaluation exact sequences shown in Fig. 1, where $i$ is an inclusion and the lower row comes from the snake lemma. The sheaves $E_{x}$ and $M$ are locally free so we see them as vector bundles of rank 3 and 4 respectively and degree -6 , so

$$
\mu\left(E_{x}\right)=-2, \quad \mu(M)=-3 / 2 .
$$

Lemma 3.2.2. The vector bundle $E_{x}$ is semi-stable.

Proof. Suppose $F$ is a subbundle of $E_{x}$. Then it is also a subbundle of $M$, but $M$ is known to be stable [EL92] because $\operatorname{deg}\left(\vartheta_{a}^{3}\right)=6$. So $\mu(F)<\mu(M)=-3 / 2$, therefore $\mu(F) \leq-2$ because $F$ is of rank 1 or 2 , i.e $\mu(F) \leq \mu\left(E_{x}\right)$. 


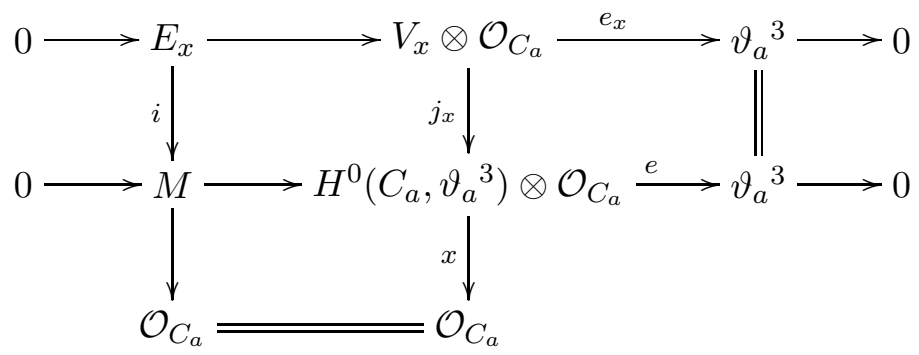

FIGURE 1. Commutative diagram 1

In particular, $E_{x}\left(\vartheta_{a}\right)$ is semi-stable (because $E_{x}$ is), of rank 3 , and has trivial determinant $\mathcal{O}_{C_{a}}$. It fits in the twisted evaluation sequence

$$
0 \rightarrow E_{x}\left(\vartheta_{a}\right) \rightarrow V_{x} \otimes \vartheta_{a} \rightarrow \vartheta_{a}^{4} \rightarrow 0
$$

We can hence define a rational map $\Psi$ from $\check{\mathbb{P}}_{a}^{4}$ to $\mathcal{S U}_{C}(3)$, regular outside of $C_{a}$ :

$$
\begin{aligned}
\Psi: \check{\mathbb{P}}_{a}^{4}-C_{a} & \rightarrow \mathcal{S U}_{C}(3) \\
x & \mapsto \Psi(x)=E_{x}\left(\vartheta_{a}\right) .
\end{aligned}
$$

We will now study this map to see that it is defined by quadrics. By Riemann-Roch, we find that there is a non trivial section $\mathcal{O}_{C_{a}} \rightarrow E_{x}\left(\omega_{C_{a}}\right)$. Since the two vector bundles are of degree 0 , this morphism is injective and the quotient is also a vector bundle. So when we twist by $\vartheta_{a} \otimes \omega_{C_{a}}^{-1}$, we obtain the following short exact sequence of vector bundles, all of degree 0:

$$
0 \rightarrow \vartheta_{a} \otimes \omega_{C_{a}}^{-1} \rightarrow E_{x}\left(\vartheta_{a}\right) \rightarrow G \rightarrow 0
$$

It follows that $E_{x}\left(\vartheta_{a}\right) \sim_{S}\left(\vartheta_{a} \otimes \omega_{C_{a}}^{-1}\right) \oplus G$, therefore

$$
\operatorname{det} G=\omega_{C_{a}} \otimes \vartheta_{a}^{-1} \quad \text { and } \quad \nu(G)=E_{x}\left(\vartheta_{a}\right) .
$$

But $\mathcal{S U}_{C}\left(2, \omega_{C_{a}} \otimes \vartheta_{a}^{-1}\right)$ sits naturally in $\mathcal{U}_{C}(2,0)$ as a fiber of the determinant map (see Lemma 3.1.1). Moreover, it is easy to check that

$$
\left.\nu\right|_{\mathcal{S U}_{C}\left(2, \omega_{C_{a}} \otimes \vartheta_{a}^{-1}\right)}: \mathcal{S U}_{C}\left(2, \omega_{C_{a}} \otimes \vartheta_{a}^{-1}\right) \rightarrow \Sigma^{\prime} \subset \mathcal{S \mathcal { U } _ { C }}(3)
$$

is isomorphic onto its image and that the composition

$$
\Phi_{3} \circ \nu: \mathcal{S U}_{C}\left(2, \omega_{C_{a}} \otimes \vartheta_{a}^{-1}\right) \rightarrow \Sigma^{\prime} \rightarrow \Sigma \subset|3 \Theta|=\mathbb{P}^{8}
$$

embeds $\mathcal{S U}_{C}\left(2, \omega_{C_{a}} \otimes \vartheta_{a}^{-1}\right)$ as a linear subspace of $|3 \Theta|$. We hence write $\mathbb{P}_{a}^{3}$ for $\mathcal{S U}_{C}\left(2, \omega_{C_{a}} \otimes\right.$ $\left.\vartheta_{a}^{-1}\right)$ and see it as a subspace of $\Sigma$ or $\Sigma^{\prime}$ interchangeably. Thus we have just proved that the map $\Psi$ actually lands into $\mathbb{P}_{a}^{3}$.

Proposition 3.2.3. The rational map $\Psi: \check{\mathbb{P}}_{a}^{4} \rightarrow \mathbb{P}_{a}^{3}$ of $(14)$ is given by a linear system of quadrics.

Proof. The degree of the linear system defining $\Psi$ is the degree of $\Psi^{*}\left(\Theta^{\text {gen }}\right)$. At the divisorial level, if we fix $L \in J^{1}$, this is just

$$
\Psi^{*}\left(\Delta_{L}\right)=\left\{x \in \check{\mathbb{P}}_{a}^{4}: H^{0}\left(C_{a}, E_{x}\left(\vartheta_{a}\right) \otimes L\right) \neq 0\right\}
$$

where $E_{x}\left(\vartheta_{a}\right)=\Psi(x)$. Let us choose $L \in J^{1}$ so that $\vartheta_{a} \otimes L$ is globally generated. By Riemann-Roch, it is easy to see that many such $L$ exist. If we twist the commutative exact diagram of Fig. 1 by $\vartheta_{a} \otimes L$, we get the commutative "long exact" diagram of Fig. 2 where 


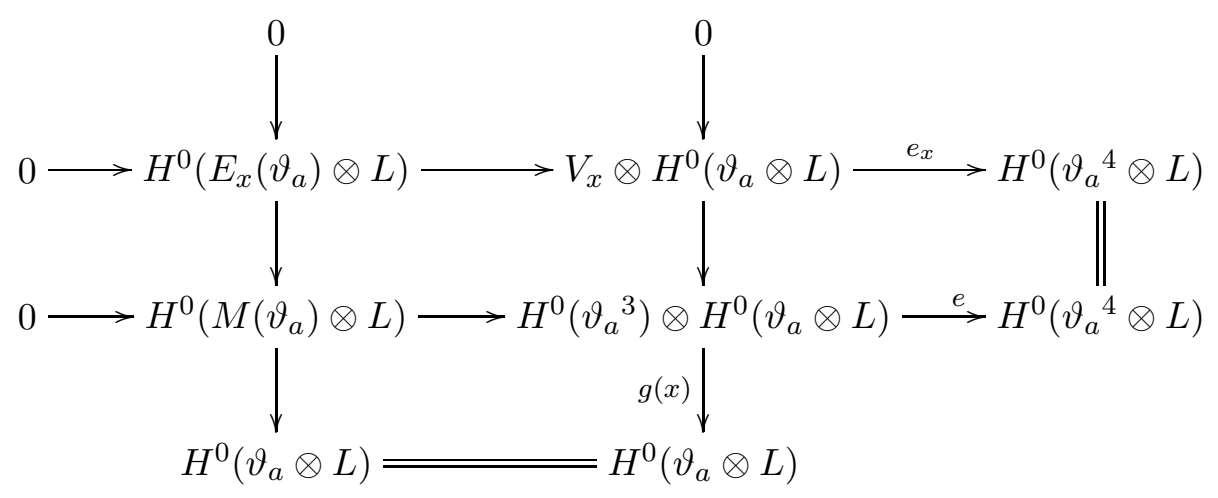

FIGURE 2. Commutative diagram 2

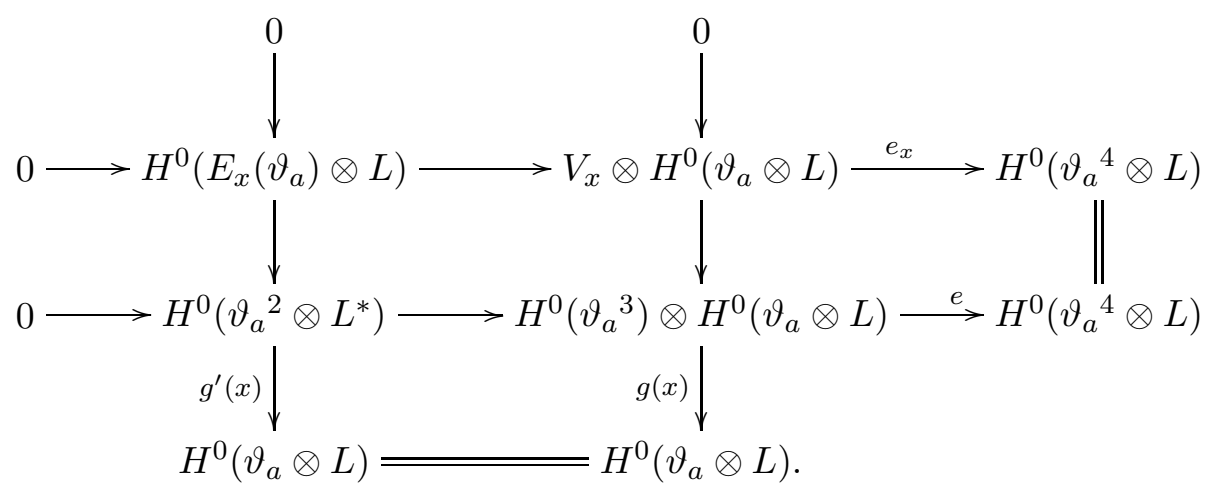

FIGURE 3. Commutative diagram 3

the cohomology groups are taken over $C_{a}$ and where the map $g(x)$ can be described as follows. The contraction

$$
H^{0}\left(\vartheta_{a}^{3}\right) \otimes H^{0}\left(\vartheta_{a}^{3}\right)^{*} \otimes H^{0}\left(\vartheta_{a} \otimes L\right) \rightarrow H^{0}\left(\vartheta_{a} \otimes L\right)
$$

defines a linear map

$$
g: H^{0}\left(\vartheta_{a}^{3}\right)^{*} \rightarrow \operatorname{Hom}\left(H^{0}\left(\vartheta_{a}^{3}\right) \otimes H^{0}\left(\vartheta_{a} \otimes L\right), H^{0}\left(\vartheta_{a} \otimes L\right)\right) .
$$

We will abuse notation and write $x$ for both an element of $\mathbb{P} H^{0}\left(\vartheta_{a}^{3}\right)^{*}$ and any of its representatives in $H^{0}\left(\vartheta_{a}^{3}\right)^{*}$. So for all $x \in H^{0}\left(\vartheta_{a}^{3}\right)^{*}$,

$$
\begin{gathered}
g(x): H^{0}\left(\vartheta_{a}^{3}\right) \otimes H^{0}\left(\vartheta_{a} \otimes L\right) \rightarrow H^{0}\left(\vartheta_{a} \otimes L\right), \quad \text { and } \\
\operatorname{ker}(g(x))=V_{x} \otimes H^{0}\left(\vartheta_{a} \otimes L\right) .
\end{gathered}
$$

Since $\operatorname{dim}\left(V_{x} \otimes H^{0}\left(\vartheta_{a} \otimes L\right)\right)=\operatorname{dim}\left(H^{0}\left(\vartheta_{a}^{4} \otimes L\right)\right)=8$, we have exactly

$$
\Psi^{*}\left(\Delta_{L}\right)=\left\{x \in \check{\mathbb{P}}_{a}^{4}: \text { the map } e_{x} \text { degenerates }\right\} .
$$

Moreover $h^{0}\left(\vartheta_{a} \otimes L\right)=2$ and $\vartheta_{a} \otimes L$ is globally generated, so by the base point free pencil trick, $\operatorname{dim} \operatorname{ker}(e)=h^{0}\left(\vartheta_{a}^{2} \otimes L^{*}\right)=2$. Therefore by restricting $g$, we get

$$
g^{\prime}: H^{0}\left(\vartheta_{a}^{3}\right)^{*} \rightarrow \operatorname{Hom}\left(H^{0}\left(\vartheta_{a}^{2} \otimes L^{*}\right), H^{0}\left(\vartheta_{a} \otimes L\right)\right) \cong \operatorname{Hom}\left(\mathbb{C}^{2}, \mathbb{C}^{2}\right),
$$

and rewrite the commutative exact diagram of Fig. 2 as that of Fig. 3. It is then clear that $e_{x}$ 
degenerates, i.e. $H^{0}\left(E_{x}\left(\vartheta_{a}\right) \otimes L\right) \neq 0$, exactly when $g^{\prime}(x)$ degenerates, since $h^{0}\left(\vartheta_{a}^{2} \otimes L^{*}\right)=$ $h^{0}\left(\vartheta_{a} \otimes L\right)=2$. So

$$
\Psi^{*}\left(\Delta_{L}\right)=\left\{x \in \mathbb{P} H^{0}\left(\vartheta_{a}^{3}\right)^{*}: \operatorname{det}\left(g^{\prime}(x)\right)=0\right\},
$$

is a quadric as it is the pull-back under $g^{\prime}$ of the discriminant locus of

$$
\operatorname{Hom}\left(H^{0}\left(\vartheta_{a}^{2} \otimes L^{*}\right), H^{0}\left(\vartheta_{a} \otimes L\right)\right) .
$$

3.3. Restriction of the dual map. Now that we have proved that the map $\Psi$ is given by quadrics, we will show that it is actually the restriction of the dual map $\mathcal{D}: \mathcal{C}_{3}-\rightarrow|3 \Theta|$.

Definition 3.3.1. The dual variety of $\mathcal{C}_{3}$, denoted $\check{\mathcal{C}}_{3}$, is the image of the dual map $\mathcal{D}$. We also call the dual map the rational map on $\check{\mathbb{P}}^{8}$. By abuse of notation, $\mathcal{D}$ denotes both the map $\mathcal{C}_{3} \rightarrow \mathbb{P}^{8}$ and the map $\check{\mathbb{P}}^{8} \rightarrow \mathbb{P}^{8}$.

By definition, the dual map or polar map $\mathcal{D}$ is given by 9 quadrics passing through the singular locus of the Coble cubic $\mathcal{C}_{3}$. The dimension count (2) shows that $\mathcal{D}$ is given by the complete linear system $\left|\mathcal{I}_{J^{1}}(2)\right|$. So when restricted to $\check{\mathbb{P}}_{a}^{4}$, for $a \in J, \mathcal{D}$ is given by quadrics in $\check{\mathbb{P}}_{a}^{4}$ containing $C_{a}=\Theta+a \subset J^{1}$.

Proposition 3.3.2. The restriction of the dual map $\left.\mathcal{D}\right|_{\check{\mathbb{P}}_{a}^{4}}: \check{\mathbb{P}}_{a}^{4} \rightarrow|3 \Theta|$ is given by the complete linear system $\left|\mathcal{I}_{C_{a}}(2)\right| \cong \mathbb{P}^{3}$ of quadrics in $\check{\mathbb{P}}_{a}^{4}$ containing $C_{a}$.

Proof. Twisting a usual sequence by $\mathcal{O}_{\check{\mathbb{P}}_{a}^{4}}(2)$, we get the exact sequence

$$
0 \rightarrow \mathcal{I}_{C_{a}}(2) \rightarrow \mathcal{O}_{\check{\mathbb{P}}_{a}^{4}}(2) \rightarrow \mathcal{O}_{C_{a}}\left(\vartheta_{a}^{6}\right) \rightarrow 0
$$

Since $C_{a}$ is of genus $2, \mathcal{I}_{C_{a}}$ is 3-regular [GLP83, Theorem 2.1], so $h^{0}\left(\check{\mathbb{P}}_{a}^{4}, \mathcal{I}_{C_{a}}(2)\right)=4$ by (16). Let $\alpha:\left|\mathcal{I}_{J^{1}}(2)\right| \rightarrow\left|\mathcal{I}_{C_{a}}(2)\right|$ be the natural restriction map. The proposition is equivalent to $\alpha$ being surjective. By contradiction, let us assume that the rank of $\alpha$ is less than 4. So we can choose a basis of the 9-dimensional space $H^{0}\left(\check{\mathbb{P}}^{8}, \mathcal{I}_{J^{1}}(2)\right)$ that consists of at least 6 quadrics that contain $\check{\mathbb{P}}_{a}^{4}$ and at most 3 quadrics that do not. Let $\mathcal{B}$ be the base locus restricted to $\check{\mathbb{P}}_{a}^{4}$ of the latter 3 quadrics: $\operatorname{dim} \mathcal{B} \geq 1$. The base locus of $H^{0}\left(\check{\mathbb{P}}^{8}, \mathcal{I}_{J^{1}}(2)\right)$, by definition exactly $J^{1}$, must then contain $\mathcal{B}$. But $J^{1} \cap \check{\mathbb{P}}_{a}^{4}=C_{a}$ is a curve of degree 6 in $\check{\mathbb{P}}_{a}^{4}$. However, $C_{a} \supseteq \mathcal{B}$ only if $\mathcal{B}$ has dimension 1 , in which case $\operatorname{deg}(\mathcal{B})=8$ : a contradiction.

Proposition 3.3.3. The rational maps $\Psi$ and $\left.\mathcal{D}\right|_{\check{\mathbb{P}}_{a}^{4}}$ are equal.

Proof. By definition, $\Psi$ is regular outside of $C_{a}$, so its base locus $B$ is contained in $C_{a}$. For the reverse inclusion, we want to show that $g^{\prime}(x)$ never has maximal rank for all $x \in C_{a}$. Elements of $H^{0}\left(C_{a}, \vartheta_{a}^{2} \otimes L^{*}\right)$ are linear combinations of tensors $s \otimes \sigma \in H^{0}\left(C_{a}, \vartheta_{a}^{3}\right) \otimes$ $H^{0}\left(C_{a}, \vartheta_{a} \otimes L\right)$ such that $s \cdot \sigma \in H^{0}\left(C_{a}, \vartheta_{a}^{4} \otimes L\right)$ is zero (see Fig. 3). But on simple tensors, $g^{\prime}(x)$ acts by

$$
g^{\prime}(x): H^{0}\left(C_{a}, \vartheta_{a}^{2} \otimes L^{*}\right) \rightarrow H^{0}\left(C_{a}, \vartheta_{a} \otimes L\right), s \otimes \sigma \mapsto s(x) \cdot \sigma,
$$

therefore the image of $g^{\prime}(x)$ is the subspace of sections in $H^{0}\left(C_{a}, \vartheta_{a} \otimes L\right)$ vanishing at $x$. However, $\vartheta_{a} \otimes L$ was assumed to be globally generated (see proof of Proposition 3.2.3), so the image of $g^{\prime}(x)$ is a proper subspace, i.e. $g^{\prime}(x)$ degenerates. Thus $B=C_{a}$. So $\Psi$ is given by a linear subseries of $\left|\mathcal{I}_{C_{a}}(2)\right|$ that has to be of dimension 3 by Proposition 3.2.3. But $\left.\mathcal{D}\right|_{\check{\mathbb{P}}_{a}^{4}}$ is given by the complete linear series $\left|\mathcal{I}_{C_{a}}(2)\right|$ which also has dimension 3 . So they are equal. 
One natural question about the construction of $\Psi$ is: what if we see $x \in \check{\mathbb{P}}_{a}^{4}$ as an element of $\check{\mathbb{P}}_{b}^{4}$, another $\mathbb{P}^{4}$ ? Since $\Psi$ (or rather $\Psi_{a}$ ) coincides with $\left.\mathcal{D}\right|_{\mathbb{P}_{a}^{4}}$, it does not matter which $\mathbb{P}^{4}$ we choose to define $\Psi(x)$ and we can extend the definition of $\Psi$ to the variety $\mathcal{W}=\bigcup_{a \in J} \check{\mathbb{P}}_{a}^{4}$. We denote the extended map $\bar{\Psi}$.

Proposition 3.3.4. We have a "global" equality of dominant rational maps:

$$
\bar{\Psi}=\left.\mathcal{D}\right|_{\mathcal{W}}: \mathcal{W} \rightarrow \Sigma=\operatorname{Sing}\left(\mathcal{C}_{6}\right)
$$

Furthermore, $\Sigma=\operatorname{Sing}\left(\mathcal{C}_{6}\right) \subset \operatorname{Sing}\left(\check{\mathcal{C}}_{3}\right)$.

Proof. The equality holds as we already know. So what is the image of $\bar{\Psi}$ ? First, the image of $\left.\mathcal{D}\right|_{\check{\mathbb{P}}_{a}^{4}}$ is the projective space $\left|\mathcal{I}_{C_{a}}(2)\right|^{*}$. Indeed a general point of $\left|\mathcal{I}_{C_{a}}(2)\right|^{*}$ corresponds to a net of quadrics in $\check{\mathbb{P}}_{a}^{4}$ whose base locus is a degree- 8 curve which can therefore contain $C_{a}$ (of degree 6). The residual curve, i.e. the general fiber of $\left.\mathcal{D}\right|_{\check{\mathbb{P}}_{a}^{4}}$, is hence a conic. So from the point of view of $\Psi$, the image of $\bar{\Psi}$ is

$$
\bigcup_{a \in J} \mathbb{P}_{a}^{3}=\Phi_{3} \circ \nu\left(\mathcal{U}_{C}(2,0)\right)=\Sigma \text {. }
$$

Furthermore, $\mathcal{W}$ is a subvariety of $\mathcal{C}_{3}$ by Proposition 3.2.1 which maps onto $\Sigma$ generically forcing one-dimensional conic fibers. By a standard property of dual varieties, if some subvariety of $\mathcal{C}_{3}$ not contained in the singular locus $J^{1}(C)$ is mapped onto a lower dimensional subvariety, then that image lies in the indeterminacy locus of the inverse dual map, i.e. in the singular locus of the dual variety $\check{\mathcal{C}}_{3}$.

\subsection{Finishing the proof of the duality and non-abelian Torelli.}

Theorem 3.4.1. The Coble hypersurfaces $\mathcal{C}_{3}$ and $\mathcal{C}_{6}$ are dual.

First, we compute the degree of $\check{\mathcal{C}}_{3}$ and state a general auxiliary lemma.

Proposition 3.4.2. The dual variety $\check{\mathcal{C}}_{3}$ is a hypersurface of degree 6 in $\mathbb{P}^{8}$.

Proof. A simple Chern class computation shows that the total degree of the dual map $\mathcal{D}$ is 6 [O005]. So if we write $d$ for the generic degree of $\mathcal{D}$, we have $d \cdot \operatorname{deg}\left(\check{\mathcal{C}}_{3}\right)=6$. To conclude that the degree of the variety has to be 6 , we use results of Section 4 that, critically, do not rely on the duality we want to prove. We know that the intersection of $\mathcal{C}_{3}$ with $\check{\mathbb{P}}_{+}^{4}(17)$ is the Segre cubic $\mathcal{S}_{3}$ (see Section 4.3). By the commutativity of (23), the proper intersection $\check{\mathcal{C}}_{3} \cap \mathbb{P}_{+}^{4}$ contains the dual variety of $\mathcal{S}_{3}$, which is known to be the Igusa-Segre quartic $\mathcal{I}_{4}$. Therefore the degree of $\check{\mathcal{C}}_{3}$ is greater than or equal to 4 . So the only possibility is 6 .

Lemma 3.4.3. Let $\mathcal{G}$ be a group acting on the projective space $\mathbb{P}^{n}$. Let $V_{d}$ be a $\mathcal{G}$-invariant hypersurface of degree $d$. Let $W_{k}$ be a hypersurface of degree $k$ such that the scheme-theoretic intersection $Y=V_{d} \cap W_{k}$ is $\mathcal{G}$-invariant. If $k<d$, then $W_{k}$ is $\mathcal{G}$-invariant.

Proof. Let $F_{d}$ (resp. $G_{k}$ ) be a homogeneous polynomial defining $V_{d}$ (resp. $W_{k}$ ). Then the homogeneous ideal of $Y$ is generated by $F_{d}$ and $G_{k}$. This ideal is $\mathcal{G}$-invariant, by this we mean that each homogeneous part is an invariant subspace of the vector space of homogeneous polynomials of fixed degree. If $k<d$, then $G_{k}$ is the only form of degree $k$, so it has to be $\mathcal{G}$-invariant.

Proof of Theorem 3.4.1 Let us assume that $\mathcal{C}_{6}$ and $\check{\mathcal{C}}_{3}$ are different. We write $Y=\mathcal{C}_{6} \cap \check{\mathcal{C}}_{3}$. Since $\mathcal{C}_{6}$ has a singular locus of codimension 2, it is irreducible. Similarly $\mathcal{C}_{3}$ is irreducible and so is its dual variety $\breve{\mathcal{C}}_{3}$ [GKZ94, Proposition 1.3]. Therefore, $Y$ is connected [FH79, Proposition 1]. We must then consider two cases. 
Case 1: $Y$ has a reduced component. If we intersect the objects with a general $\mathbb{P}^{3}$, we see that the surface $S=\mathcal{C}_{6} \cap \mathbb{P}^{3}$ has $45 A_{1}$-singularities, according to the local analytic description of $\Sigma^{\prime} \subseteq \mathcal{S U}_{C}(3)$ in [Las96] and the fact that $\operatorname{deg}(\Sigma)=45$. Since $T=\check{\mathcal{C}}_{3} \cap$ $\mathbb{P}^{3}$ is different from $S$, we denote by $D$ the Cartier divisor of $S$ defined by the complete intersection with $T$. It is a connected curve with a reduced component. We resolve the 45 rational double points and get 45 exceptional (-2)-curves $E_{1}, \ldots, E_{45}$ in the resolution $\pi: \tilde{S} \rightarrow S$. Now let $H$ be the pullback of the hyperplane section of $S$. Its self-intersection in $\tilde{S}$ is $\operatorname{deg}_{\tilde{S}}\left(H^{2}\right)=6$. Since $T$ is also singular at those 45 points by Proposition 3.3 .4 , the proper transform $\tilde{D}$ of $D$ under the blowup map $\pi$ is linearly equivalent to

$$
\tilde{D}=6 H-\sum_{i=1}^{45} a_{i} E_{i}, \quad a_{i} \geq 2 .
$$

But $\pi$ is a crepant resolution, so

$$
\omega_{\tilde{S}}=\pi^{*}\left(\omega_{\mathbb{P}^{3}} \otimes \mathcal{O}_{\mathbb{P}^{3}}(S) \otimes \mathcal{O}_{S}\right)=\mathcal{O}(2 H) .
$$

We can also compute the arithmetic genus $p_{a}(\tilde{D})$ of $\tilde{D}$, knowing that $\tilde{D}$ is reduced:

$$
2 p_{a}(\tilde{D})-2=\operatorname{deg}_{\tilde{S}}\left(\left(K_{\tilde{S}}+\tilde{D}\right) \cdot \tilde{D}\right)=288+\sum_{i=1}^{45}(-2) a_{i}^{2} \leq 288-360=-72,
$$

because $a_{i} \geq 2$. Therefore we see that $p_{a}(\tilde{D}) \leq-35$, which is not possible, since $\tilde{D}$ is an effective reduced connected Cartier divisor on a nonsingular surface. So $S=T$. Moreover, since the intersecting $\mathbb{P}^{3}$ was general, $\mathcal{C}_{6}=\check{\mathcal{C}}_{3}$.

Case 2: $Y$ has no reduced components. We write the decomposition of $Y$ into irreducible components:

$$
Y=a_{1} Y_{1}+a_{2} Y_{2}+\cdots+a_{m} Y_{m}
$$

where $a_{i} \geq 2$ and $Y_{i}$ are prime Cartier divisors on $\mathcal{C}_{6}$ of respective degrees $d_{i}$. But $Y$ is of degree 36 , therefore $a_{1} d_{1}+\cdots+a_{m} d_{m}=36$. Since $\operatorname{dim} \mathcal{C}_{6} \geq 3$, we know by a Lefschetz-type Theorem ([Gro68, Exposé XII, Corollary 3.7]) that the restriction map Pic $\mathbb{P}^{8} \stackrel{\sim}{\rightarrow}$ Pic $\mathcal{C}_{6}$ is an isomorphism. So each prime divisor $Y_{i}$ is cut out by a hypersurface in $\mathbb{P}^{8}$ and it follows that 6 divides $d_{i}$. Thus the only possible cases are

- $m=1:\left(a_{1}, d_{1}\right)=(2,18)$ or $(3,12)$ or $(6,6)$.

- $m=2:\left\{\left(a_{1}, d_{1}\right),\left(a_{2}, d_{2}\right)\right\}=\{(2,12),(2,6)\}$ or $\{(3,6),(3,6)\}$.

- $m=3:\left\{\left(a_{1}, d_{1}\right),\left(a_{2}, d_{2}\right)\left(a_{3}, d_{3}\right)\right\}=\{(2,6),(2,6),(2,6)\}$.

In every case, we can see that the $a_{i}$ have a common divisor that is either 2,3 or 6 . So we can rewrite

$$
Y=2 Z \text { or } 3 Z \text { or } 6 Z \text {. }
$$

A contradiction will arise from the $J_{3}$-invariance of $Y$. We already know that $\mathcal{C}_{6}$ and $\mathcal{C}_{3}$ are $J_{3}$-invariant (Proposition 2.1.2). Moreover, it is easy to see that $\mathcal{D}$ is $J_{3}$-equivariant directly from the partial derivatives of (5). Therefore $\check{\mathcal{C}}_{3}$ is $J_{3}$-invariant, and so is $Y$ and then $Z$. If $Y=3 Z$ or $6 Z$, then $Z$ is a quadric section or a hyperplane section, but there are no $J_{3}$-invariant quadrics or hyperplanes in $\check{\mathbb{P}}^{8}$. Thus by Lemma 3.4.3, we get a contradiction. So we are left with the case $Y=2 Z$, and $Z$ is cut out by a $J_{3}$-invariant cubic. Again, by Proposition 3.3.4, we know that $\Sigma \subset Z$. We intersect with the fixed $\mathbb{P}_{+}^{4}$ (6), so $Z \cap \mathbb{P}_{+}^{4}$ must contain $\Sigma \cap \mathbb{P}_{+}^{4}$. But as a consequence of Theorem 2.2.1, we know that [NR03, Eq. (5.2)]

$$
\Sigma \cap \mathbb{P}_{+}^{4}=2 H \cup\left\{\left(15_{3}\right) \text {-configuration of lines and points }\right\},
$$

where $H$ is a hyperplane of $\mathbb{P}_{+}^{4}$. The configuration of lines and points does not lie in a hyperplane, so $\Sigma \cap \mathbb{P}_{+}^{4}$ cannot lie in a cubic hypersurface of $\mathbb{P}_{+}^{4}$, which shows that $\mathbb{P}_{+}^{4}$ must 
lie in the $J_{3}$-invariant cubic hypersurface cutting out $Z$. However, by Proposition $4.3 .1, \mathbb{P}_{+}^{4}$ cannot be contained in a $J_{3}$-invariant cubic. We hence ruled out all the possibilities.

The established duality will be used in the next section to recover some classical dualities and reinterpret them in terms of vector bundles. But an easy corollary is the following nonabelian Torelli theorem:

Corollary 3.4.4 (Non-abelian Torelli Theorem). Let $C$ and $C^{\prime}$ be two smooth projective curves of genus two. If $\mathcal{S U}_{C}(3)$ is isomorphic to $\mathcal{S U}_{C^{\prime}}(3)$, then $C$ is isomorphic to $C^{\prime}$.

Proof. Starting from $\mathcal{S U}_{C}(3)$, there is a canonical way to retrieve $C$. We first take the ample generator $\Theta^{\text {gen }}$ of $\operatorname{Pic}\left(\mathcal{S U}_{C}(3)\right)$, look at the map associated to the line bundle. The branch locus of the 2-1 map has dual variety a cubic hypersurface in $\mathbb{P}^{8}$ singular exactly along the principally polarized Jacobian $\left(J^{1}(C), \Theta\right)$, which determines $C$ by the usual Torelli theorem.

Remark 3.4.5. The non-abelian Torelli question has been raised ever since the construction of the moduli spaces $\mathcal{S U}_{C}(r, d)(g \geq 2)$. In [MN68], Mumford and Newstead prove the theorem in the case of rank 2 , odd degree determinant $(d=1)$, and $g \geq 2$. It is further generalized in [NR75] and [Tyu74] to all non trivial smooth cases $((r, d)=1)$. Then Balaji proves the theorem for $r=2, d=0$ on a curve of genus $g \geq 3$ [Bal90], before Kouvidakis and Pantev extend the result to any $r$ and $d$, still for $g \geq 3$ [KP95].

In [HR04], Hwang and Ramanan introduce a stronger non-abelian Torelli result. If we denote by $\mathcal{S U}_{C}(r, d)^{s}$ the moduli space of stable vector bundles, which is open in $\mathcal{S U}_{C}(r, d)$, then $\mathcal{S U}_{C}(r, d)^{s} \cong \mathcal{S U}_{C^{\prime}}(r, d)^{s}$ implies that $C \cong C^{\prime}$, and this for any $r$ and $d$, but for $g \geq 4$. Our version of non-abelian Torelli is a new case and it can also be shown to be strong by adapting the same argument.

\section{The Geometry of the FiXed Loci}

In this section, we focus our attention to the dual maps:

$$
\mathcal{C}_{6} \subset \mathbb{P}^{8} ₹=-\frac{\mathcal{D}^{\prime}}{-}-\overline{\mathcal{D}}- \pm \check{\mathbb{P}^{8}} \supset \mathcal{C}_{3},
$$

especially, these dual maps restricted to the fixed loci $\operatorname{Fix}(\tau)$ defined in (6):

$$
\operatorname{Fix}(\tau)=\mathbb{P}_{+}^{4} \sqcup \mathbb{P}_{-}^{3} \subset|3 \Theta|=\mathbb{P}^{8}, \quad \text { and } \quad \operatorname{Fix}(\tau)=\check{\mathbb{P}}_{+}^{4} \sqcup \check{\mathbb{P}}_{-}^{3} \subset|3 \Theta|^{*}=\check{\mathbb{P}}^{8} .
$$

Notation. We use the following convention, based on the domain space. For all dual maps (i.e. $\mathcal{D}, \mathcal{D}^{\prime}$, and their restrictions), the apostrophe means that the domain is or lies in $\mathbb{P}^{8} \cong|3 \Theta|$ and not $\check{\mathbb{P}}^{8}$. The use of the subscripts + or - determines to which fix locus we restrict the maps. For instance, $d_{-}^{\prime}$ will denote the restriction $\left.\mathcal{D}^{\prime}\right|_{\mathbb{P}_{-}^{3}}: \mathbb{P}_{-}^{3} \rightarrow \check{\mathbb{P}}^{8}$. Also, maps that carry ${ }^{\sim}$ are maps that stay in $\check{\mathbb{P}}^{8}$.

4.1. $\tau$-equivariance and target spaces. In order to study the dual maps $\mathcal{D}$ and $\mathcal{D}^{\prime}$, we will verify that they are $\tau$-equivariant. Although it is not the most intrinsic way to proceed, we will use an explicit basis for the vector spaces $H^{0}\left(J^{1}, \mathcal{O}(3 \Theta)\right)$ and its dual. This amounts to choosing a level-3 structure on the Jacobian $J$, just as in Theorem 2.1.1. The basis is $\left\{e_{b}\right\}_{b \in\left(\mathbb{F}_{3}\right)^{2}}$ and the corresponding coordinate system $\left\{X_{b}\right\}_{b \in\left(\mathbb{F}_{3}\right)^{2}}$.

Proposition 4.1.1. (i) The Coble hypersurfaces $\mathcal{C}_{3}$ and $\mathcal{C}_{6}$ are $\tau$-invariants. Moreover, the polynomials defining $\mathcal{C}_{3}$ and $\mathcal{C}_{6}$ are $\tau$-invariant. 
(ii) The dual maps $\mathcal{D}$ and $\mathcal{D}^{\prime}$ are $\tau$-equivariant. So the fixed loci are mapped to the fixed loci by the dual maps.

Proof. (i) For $\mathcal{C}_{3}$, this is clear from the equation (5) and the fact that $\tau \cdot X_{b}=X_{-b}$. For $\mathcal{C}_{6}$, let $E \in \mathcal{S U}_{C}(3)$, so $\tau^{\prime}(E)=E^{*}$. If $E \in \mathcal{C}_{6}$ (i.e. $h^{*} E^{*}=E$ ), then $h^{*}\left(\tau^{\prime} E\right)^{*}=h^{*} E=$ $E^{*}=\tau^{\prime}(E)$, which means $\tau^{\prime}(E) \in \mathcal{C}_{6}$. So $\mathcal{C}_{6}$ is $\tau^{\prime}$-invariant as a ramification locus, i.e. $\tau$-invariant as a branch locus, and the polynomial $F_{6}$ defining $\mathcal{C}_{6}$ is either $\tau$-invariant or $\tau$-anti-invariant. But if $F_{6}$ is $\tau$-anti-invariant, then it must contain $\mathbb{P}_{+}^{4}$ for if $V_{+}$is the vector space such that $\mathbb{P}_{+}^{4}=\mathbb{P}\left(V_{+}\right)$and if we take $a \in V_{+}$, then

$$
F_{6}(a)=F_{6}(\tau \cdot a) \stackrel{\text { def }}{=} \tau \cdot F_{6}(a)=-F_{6}(a) .
$$

So we see that $F_{6}(a)$ must be zero. But we know that $\mathbb{P}_{+}^{4}$ is not contained in $\mathcal{C}_{6}$ [Ngu05, Proposition III.1]. Thus $F_{6}$ is $\tau$-invariant.

(ii) An easy exercise left to the reader.

We now describe the fixed spaces: $\mathbb{P}_{-}^{3} \subset \mathbb{P}^{8}=|3 \Theta|$ is defined by the equations

$$
X_{00}=0, X_{01}+X_{02}=0, X_{10}+X_{20}=0, X_{11}+X_{22}=0, X_{12}+X_{21}=0,
$$

while $\mathbb{P}_{+}^{4}$ is defined by

$$
X_{01}-X_{02}=0, X_{10}-X_{20}=0, X_{11}-X_{22}=0, X_{12}-X_{21}=0 .
$$

Moreover, the inclusions of $\mathbb{P}_{-}^{3}$ and $\mathbb{P}_{+}^{4}$ in $\mathbb{P}^{8}$ are given by the linear injections $\gamma_{-}: \mathbb{C}^{4} \rightarrow \mathbb{C}^{9}$ and $\gamma_{+}: \mathbb{C}^{5} \rightarrow \mathbb{C}^{9}$ where

$$
\begin{aligned}
\gamma_{-}\left(Z_{0}, Z_{1}, Z_{2}, Z_{3}\right) & =\left(X_{00}, X_{01}, X_{02}, X_{10}, X_{11}, X_{12}, X_{20}, X_{21}, X_{22}\right), \\
& =\left(0, Z_{0},-Z_{0}, Z_{1}, Z_{2}, Z_{3},-Z_{1},-Z_{3},-Z_{2}\right), \quad \text { and } \\
\gamma_{+}\left(Y_{0}, Y_{1}, Y_{2}, Y_{3}, Y_{4}\right) & =\left(X_{00}, X_{01}, X_{02}, X_{10}, X_{11}, X_{12}, X_{20}, X_{21}, X_{22}\right), \\
& =\left(Y_{0}, Y_{1}, Y_{1}, Y_{2}, Y_{3}, Y_{4}, Y_{2}, Y_{4}, Y_{3}\right) .
\end{aligned}
$$

Proposition 4.1.2. (i) The dual map $\mathcal{D}: \check{\mathbb{P}}^{8} \rightarrow \mathbb{P}^{8}=|3 \Theta|$ maps the fixed loci in the following way:

$$
\mathcal{D}\left(\check{\mathbb{P}}_{+}^{4}\right) \subset \mathbb{P}_{+}^{4}, \quad \mathcal{D}\left(\check{\mathbb{P}}_{-}^{3}\right) \subset \mathbb{P}_{+}^{4} .
$$

(ii) The dual map $\mathcal{D}^{\prime}: \mathbb{P}^{8} \rightarrow \check{\mathbb{P}}^{8}=|3 \Theta|^{*}$ maps the fixed loci in the following way:

$$
\mathcal{D}^{\prime}\left(\mathbb{P}_{+}^{4}\right) \subset \check{\mathbb{P}}_{+}^{4}, \quad \mathcal{D}^{\prime}\left(\mathbb{P}_{-}^{3}\right) \subset \check{\mathbb{P}}_{-}^{3} .
$$

Proof. (i) Let $X_{00}, X_{01}, \ldots, X_{22}$ be the homogeneous coordinates of $\mathbb{P}^{8}=|3 \Theta|$ and $F$ be a homogeneous $\tau$-invariant (Proposition 4.1.1) polynomial of degree 6 defining the Coble sextic. For notational convenience, let us write for $b \in\left(\mathbb{F}_{3}\right)^{2}$

$$
F_{b}^{\prime}\left(Z_{0}, Z_{1}, Z_{2}, Z_{3}\right)=\frac{\partial F}{\partial X_{b}}\left(0, Z_{0},-Z_{0}, Z_{1}, Z_{2}, Z_{3},-Z_{1},-Z_{3},-Z_{2}\right)
$$

a homogeneous polynomial of degree 5 . So

$$
F_{b}^{\prime}\left(Z_{0}, Z_{1}, Z_{2}, Z_{3}\right)=F_{-b}^{\prime}\left(-Z_{0},-Z_{1},-Z_{2},-Z_{3}\right)=-F_{-b}^{\prime}\left(Z_{0}, Z_{1}, Z_{2}, Z_{3}\right),
$$

which proves that

$$
F_{00}^{\prime}=0, \quad F_{10}^{\prime}=-F_{20}^{\prime}, \quad F_{11}^{\prime}=-F_{22}^{\prime}, \quad \text { and } F_{12}^{\prime}=-F_{21}^{\prime} .
$$

Hence, the dual map restricted to $\mathbb{P}_{-}^{3}$ is given as follows:

$$
\begin{aligned}
\mathcal{D}^{\prime} \circ \gamma_{-}: \mathbb{P}_{-}^{3} & \longrightarrow \check{\mathbb{P}}^{8} \\
{\left[Z_{0}: Z_{1}: Z_{2}: Z_{3}\right] } & \longmapsto\left[0: F_{01}^{\prime}:-F_{01}^{\prime}: F_{10}^{\prime}: F_{11}^{\prime}: F_{12}^{\prime}:-F_{10}^{\prime}:-F_{12}^{\prime}:-F_{11}^{\prime}\right]
\end{aligned}
$$


and the image clearly lies in $\check{\mathbb{P}}_{-}^{3}$. Then similarly, for $\mathbb{P}_{+}^{4}$, if we write

$$
G_{b}^{\prime}\left(Y_{0}, Y_{1}, Y_{2}, Y_{3}, Y_{4}\right)=\frac{\partial F}{\partial X_{b}}\left(Y_{0}, Y_{1}, Y_{1}, Y_{2}, Y_{3}, Y_{4}, Y_{2}, Y_{4}, Y_{3}\right)
$$

it is easy to see that the dual map $\mathcal{D}^{\prime}$ sends $\mathbb{P}_{+}^{4}$ to $\check{\mathbb{P}}_{+}^{4}$ since

$$
G_{01}^{\prime}=G_{02}^{\prime}, \quad G_{10}^{\prime}=G_{20}^{\prime}, \quad G_{11}^{\prime}=G_{22}^{\prime}, \quad G_{12}^{\prime}=G_{21}^{\prime} .
$$

(ii) The proof is completely similar, except for the fact that the dual map $\mathcal{D}$ is given by quadrics instead of quintics, so it sends $\check{\mathbb{P}}_{-}^{3}$ to $\mathbb{P}_{+}^{4}$.

4.2. The Hexahedron and the original curve. In this section, we will study the image of the hexahedron $\mathfrak{H}$ of Theorem 2.2.2 under the dual map $\mathcal{D}^{\prime}$ and prove that it also determines our original curve $C$ of genus 2 .

Theorem 4.2.1. The 6 planes making $\mathfrak{H}$ correspond to the 6 Weierstra $\beta$ points of our original curve $C$. That is, the six planes correspond to 6 points in the dual projective space $\check{\mathbb{P}}_{-}^{3}$ of $\mathbb{P}_{-}^{3}$ through which passes a unique twisted cubic. On this rational curve, the points are projectively equivalent to the 6 Weierstraß points of our given curve $C$.

First, let us introduce more notation. Again, we use $Z_{0}, Z_{1}, Z_{2}, Z_{3}$ as the homogeneous coordinates of $\mathbb{P}_{-}^{3}$ and $F$ as a polynomial defining $\mathcal{C}_{6}$ in $\mathbb{P}^{8}$. We keep the notations of (18) and (19). Let

$$
H\left(Z_{0}, Z_{1}, Z_{2}, Z_{3}\right)=F \circ \gamma_{-}=F\left(0, Z_{0},-Z_{0}, Z_{1}, Z_{2}, Z_{3},-Z_{1},-Z_{3},-Z_{2}\right)
$$

be the equation defining the hexahedron. Let $\delta_{-}^{\prime}$ be the "restricted" dual rational map

$$
\begin{gathered}
\delta_{-}^{\prime}: \mathbb{P}_{-}^{3} \rightarrow \check{\mathbb{P}}_{-}^{3} \\
{\left[Z_{0}: \cdots: Z_{3}\right] \mapsto\left[\frac{\partial H}{\partial Z_{0}}: \cdots: \frac{\partial H}{\partial Z_{3}}\right] .}
\end{gathered}
$$

Proposition 4.2.2. Let $\check{\gamma}_{-}$be the embedding of $\check{\mathbb{P}}_{-}^{3}$ in $\check{\mathbb{P}}^{8}$. Then the following diagram commutes.

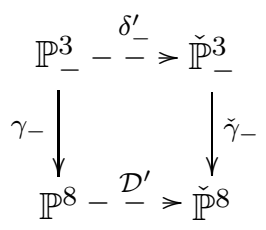

Proof. The proof is straightforward and does not depend of the duality of the Coble hypersurfaces proved in Theorem 3.4.1. By (20) and (21), we see that

$$
\mathcal{D}^{\prime} \circ \gamma_{-}=\left[0: F_{01}^{\prime}:-F_{01}^{\prime}: F_{10}^{\prime}: F_{11}^{\prime}: F_{12}^{\prime}:-F_{10}^{\prime}:-F_{12}^{\prime}:-F_{11}^{\prime}\right] \text {. }
$$

On the other hand, we compute $\partial H / \partial Z_{i}$ and find:

$$
\begin{array}{lll}
\frac{\partial H}{\partial Z_{0}}=\sum_{(i, j) \in\left(\mathbb{F}_{3}\right)^{2}} \frac{\partial F}{\partial X_{i, j}} \frac{\partial X_{i, j}}{\partial Z_{0}}=2 F_{01}^{\prime}, & & \frac{\partial H}{\partial Z_{1}}=F_{10}^{\prime}-F_{20}^{\prime}=2 F_{10}^{\prime}, \\
\frac{\partial H}{\partial Z_{2}}=F_{11}^{\prime}-F_{22}^{\prime}=2 F_{11}^{\prime}, & \frac{\partial H}{\partial Z_{3}}=F_{12}^{\prime}-F_{21}^{\prime}=2 F_{12}^{\prime} .
\end{array}
$$

It is then easy to check that the diagram commutes.

Therefore the map $\delta_{-}^{\prime}$ can be seen as the restriction of the dual map $\mathcal{D}^{\prime}$. 
Proof of Theorem 4.2.1 The restricted dual map $\delta_{-}^{\prime}$ associates to a smooth point of the hexahedron the tangent plane through it. But each plane is its own tangent plane so the planes contract to 6 distinct points in the dual $\mathbb{P}^{3}$. Moreover, each plane lies in the Coble sextic $\mathcal{C}_{6}$ and is contracted, so the six points must be in the singular locus of Coble cubic $\mathcal{C}_{3}$ restricted to $\check{\mathbb{P}}_{-}^{3}$, which consists exactly of the 6 odd theta characteristics of our original curve $C$. The double cover of the unique twisted cubic through these 6 points and branched at these 6 points is then a curve of genus 2 having these 6 points as Weierstraß points (or odd theta characteristics). So it must be isomorphic to $C$.

4.3. The Segre cubic primal and its dual. In this section we study the dual map $\mathcal{D}$ restricted to $\check{\mathbb{P}}_{+}^{4}$, which we know, by the following theorem, is not contained in the Coble cubic $\mathcal{C}_{3}$.

Proposition 4.3.1. A $\left(\mathbb{F}_{3}\right)^{4}$-invariant cubic polynomial on $\check{\mathbb{C}}^{9}$ does not vanish on $\check{\mathbb{C}}_{+}^{5}$, the vector subspace whose projectivization is $\check{\mathbb{P}}_{+}^{4}$.

Proof. The proof is easily done by looking at the equations of the $\left(\mathbb{F}_{3}\right)^{4}$-invariant cubics, which, for some constants $\alpha_{0}, \ldots, \alpha_{4}$, are exactly of the form (5). So restricted to $\check{C}_{+}^{5}$, as given by $\check{\gamma}_{+}$just like in (18), we get:

$$
\begin{aligned}
\frac{\alpha_{0}}{3}\left(Y_{0}^{3}+2 Y_{1}^{3}+\right. & \left.2 Y_{2}^{3}+2 Y_{3}^{3}+2 Y_{4}^{3}\right)+2 \alpha_{1}\left(Y_{0} Y_{1}^{2}+2 Y_{2} Y_{3} Y_{4}\right) \\
& 2 \alpha_{2}\left(Y_{0} Y_{2}^{2}+2 Y_{1} Y_{3} Y_{4}\right)+2 \alpha_{3}\left(Y_{0} Y_{3}^{2}+2 Y_{1} Y_{2} Y_{4}\right)+2 \alpha_{4}\left(Y_{0} Y_{4}^{2}+2 Y_{1} Y_{2} Y_{3}\right) .
\end{aligned}
$$

Clearly, the five terms are linearly independent.

Thus the intersection $\check{\mathbb{P}^{4}}+\cap \mathcal{C}_{3}$ is a cubic threefold with 10 nodes (the maximal number possible). It is classically known that this is only one such threefold in $\check{\mathbb{P}}_{+}^{4}$ : the Segre cubic. We write $\mathcal{S}_{3}=\check{\mathbb{P}}_{+}^{4} \cap \mathcal{C}_{3}$. Following the notations of (18) and Section 4.1, let $Y_{0}, \ldots, Y_{4}$ be the homogeneous coordinates of $\check{\mathbb{P}}_{+}^{4}$ and $G$ be a homogeneous polynomial defining the Coble cubic in $\check{\mathbb{P}}^{8}$, so that

$$
S\left(Y_{0}, Y_{1}, Y_{2}, Y_{3}, Y_{4}\right)=G \circ \check{\gamma}_{+}=G\left(Y_{0}, Y_{1}, Y_{1}, Y_{2}, Y_{3}, Y_{4}, Y_{2}, Y_{4}, Y_{3}\right)
$$

is an equation for $\mathcal{S}_{3} \subset \check{\mathbb{P}}_{+}^{4}$. Then we have the small dual map

$$
\delta_{+}: \check{\mathbb{P}}_{+}^{4} \rightarrow \mathbb{P}_{+}^{4},\left[Y_{0}: \cdots: Y_{4}\right] \mapsto\left[\frac{\partial S}{\partial Y_{0}}: \cdots: \frac{\partial S}{\partial Y_{4}}\right]
$$

Similarly to Proposition 4.2.2, we have a commutative diagram

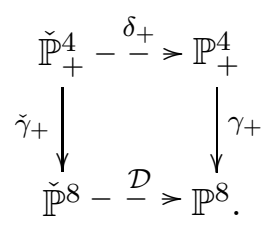

So under the global dual map $\mathcal{D}$, the Segre cubic $\mathcal{S}_{3}$ is sent to its dual variety, the IgusaSegre quartic $\mathcal{I}_{4}$, hence justifying Theorem 2.2.1 and the presence of $\mathcal{I}_{4}$. 
4.4. The Weddle quartic. In this section we study the dual map $\mathcal{D}$ restricted to $\check{\mathbb{P}}_{-}^{3}$ which is contained in the Coble cubic $\mathcal{C}_{3}$.

Using the explicit expression for the Coble cubic explicitly given in (5), we can see that the image of $\check{\mathbb{P}}_{-}^{3}$ is a $\mathbb{P}^{3}$ of which a set of equations is

$$
\begin{gathered}
\alpha_{0} X_{00}+\alpha_{1} X_{01}+\alpha_{2} X_{10}+\alpha_{3} X_{12}+\alpha_{4} X_{11}=0 \\
X_{01}-X_{02}=0, \quad X_{10}-X_{20}=0, \quad X_{11}-X_{22}=0, \quad X_{12}-X_{21}=0 .
\end{gathered}
$$

In particular, this $\mathbb{P}^{3}$ is contained in $\mathbb{P}_{+}^{4}$, which confirms Proposition 4.1.2,

Moreover, this restricted dual map, which we denote $\check{\delta}_{-}$, is given by the complete linear system (easy to check) of quadrics in $\check{\mathbb{P}}_{-}^{3}$ passing through $J^{1} \cap \mathcal{C}_{3}$, i.e. the six odd theta characteristics of $C$. Now the story is classically well known and we will not prove the following result.

Proposition 4.4.1. The rational map $\check{\delta}_{-}$is generically 2 to 1 . The ramification locus is a quartic surface $\mathcal{W}_{4}$ with nodes at the 6 points of $J^{1} \cap \mathcal{C}_{3}$, and the branch locus a quartic Kummer surface $\mathcal{B K}_{4}$.

Definition 4.4.2. The ramification locus $\mathcal{W}_{4}$ of Proposition 4.4.1 is called the Weddle quartic surface.

This surface was first identified by T. Weddle in 1850 ([Wed50] footnote p. 69). A nice treatise can be found in [Hud90] or [Cob61].

An important remark is that $\mathbb{P}^{3}=\operatorname{Im}\left(\check{\delta}_{-}\right)$lies not only in $\mathbb{P}_{+}^{4}$ but also in the Coble sextic $\mathcal{C}_{6}$ because the source space $\check{\mathbb{P}}_{-}^{3}$ lies in the Coble cubic $\mathcal{C}_{3}$. From Theorem 2.2.1, it follows that $\mathbb{P}^{3}$ is the tangent hyperplane $V_{0}$. It is then natural to guess that the Kummer surface $\mathcal{B K}_{4}$ of Proposition 4.4.1 is the same as $\mathcal{K}^{\prime}(7)$, since it sits already in $V_{0}$. More generally, the 6 points in $\check{\mathbb{P}}_{-}^{3}$ correspond to a unique curve of genus two (here it is our original $C$ ), and the Kummer surface associated to the Weddle surface defined by the 6 points should be the Kummer surface of $J(C)$. Of course this is true (see [Hud90, Bak22, Cob61]), however, we give here a proof along the lines of the original results but putting forth previously exhibited vector bundles of rank 3 .

To identify the two Kummer surfaces, we will identify the nodes and since fixing nodes determines a unique Kummer surface [Hud90], we get the desired result. For this, we adapt A. Ortega Ortega's description of the dual map on the secant variety of the embedded $J^{1}$ [0005, Section 7]. The dual map $\mathcal{D}$ is constant on secant of $J^{1}$, i.e. it contracts the secant. So the image is determined by the 2 points on $J^{1}$ defining the secant and there is a rational map

$$
\psi: J^{1} \times J^{1} \rightarrow|3 \Theta| \cong \mathbb{P}^{8}
$$

defining the dual map $\mathcal{D}$ in the following sense. Let $z \in|3 \Theta|^{*}$ be a point on the secant line passing through $a \in J^{1}$ and $b \in J^{1}$ but distinct from $a$ and $b$. Then

$$
\mathcal{D}(z)=\psi(a, b) .
$$

We first define $\psi$. Given a point $x$ on the Jacobian $J$ of the original curve $C$, we denote by $\Theta_{x}$ the translate $t_{x}^{*} \Theta$ of the canonical Riemann theta divisor of $J^{1}$. If we fix two distinct points $a, b \in J^{1}$, then there exist $x, y \in J$ such that $\Theta_{x} \cap \Theta_{y}=\{a, b\}$, and we set

$$
\psi(a, b)=\Theta_{x}+\Theta_{y}+\Theta_{-x-y} \in|3 \Theta| .
$$

We now extend Ortega Ortega's result by showing that the divisor $\Theta_{x}+\Theta_{y}+\Theta_{-x-y}$ correspond to the vector bundle $x \oplus y \oplus\left(x^{-1} \otimes y^{-1}\right)$. Indeed, under the double cover map 
$\Phi_{3}$ (1), the vector bundle $x \oplus y \oplus\left(x^{-1} \otimes y^{-1}\right)$ goes to the divisor

$$
\begin{aligned}
D_{x \oplus y \oplus\left(x^{-1} \otimes y^{-1}\right)} & =\left\{L \in J^{1}: h^{0}\left(C,(L \otimes x) \oplus(L \otimes y) \oplus\left(L \otimes x^{-1} \otimes y^{-1}\right)\right)>0\right\}, \\
& =\Theta_{x}+\Theta_{y}+\Theta_{-x-y} .
\end{aligned}
$$

So we have proved:

Lemma 4.4.3. The dual map $\mathcal{D}$ is defined on secants of $J^{1}$ by the rational map

$$
\begin{aligned}
\psi: J^{1} \times J^{1} & \longrightarrow \operatorname{Sing}\left(\mathcal{S U}_{C}(3)\right) \\
(a, b) & \longmapsto x \oplus y \oplus\left(x^{-1} \otimes y^{-1}\right)
\end{aligned}
$$

where $x, y \in J$ are such that $\Theta_{x} \cap \Theta_{y}=\{a, b\}$.

Proposition 4.4.4. The branch locus $\mathcal{B K}_{4}$ of the map rational map $\check{\delta}_{-}$is the Kummer surface $\mathcal{K}^{\prime}=\left\{\mathcal{O}_{C} \oplus x \oplus x^{-1}: x \in J\right\}$, i.e. the intersection of $V_{0}$ with $\mathcal{I}_{4}$.

Proof. Let us apply Lemma 4.4.3. We know classically that the 15 secants joining any two points of $P$, the set of odd theta characteristics, are contracted to nodes of the Kummer surface $\mathcal{B K}_{4}$. Let $a, b \in J^{1}$ be two odd theta characteristics. We form the 2-torsion point $\epsilon=a-b \in J_{2}$, and denote by $p_{\epsilon}$ the node of $\mathcal{B} \mathcal{K}_{4}$ resulting from the contraction of the chord joining $a$ and $b$. By definition,

$$
\Theta \cap \Theta_{\epsilon}=\left\{L \in J^{1}: h^{0}(C, L)>0 \text { and } h^{0}(C, L \otimes \epsilon)>0\right\} .
$$

We now check that $a$ and $b$ are in the intersection, therefore proving that the nodes $p_{\epsilon}$ correspond to vector bundles of the form $\mathcal{O}_{C} \oplus \epsilon \oplus \epsilon$, which are nodes of $\mathcal{K}^{\prime}$. Indeed,

$$
\begin{array}{ll}
h^{0}\left(C, \mathcal{O}_{C}(a)\right)>0 & h^{0}\left(C, \mathcal{O}_{C}(a+a-b)\right)=h^{0}\left(C, \mathcal{O}_{C}(b)\right)>0, \\
h^{0}\left(C, \mathcal{O}_{C}(b)\right)>0 & h^{0}\left(C, \mathcal{O}_{C}(b+a-b)\right)=h^{0}\left(C, \mathcal{O}_{C}(a)\right)>0 .
\end{array}
$$

Thus we have proved that the Kummer surfaces $\mathcal{B} \mathcal{K}_{4}$ and $\mathcal{K}^{\prime}$ share 15 nodes. Their wellknown configuration guarantees that there is a conic on which 6 of the 15 nodes lie. The double cover of this curve, branched over the 6 points, is a curve of genus two, whose Jacobian is the unique (up to isomorphism) abelian surface covering the Kummer surfaces. So the Kummer surfaces $\mathcal{B K}_{4}$ and $\mathcal{K}^{\prime}$ are isomorphic and the curve of genus two is isomorphic to our original curve $C$, since $\mathcal{K}^{\prime}$ is the Kummer surface associated to $C$. Moreover, $\mathcal{B K}_{4}$ and $\mathcal{K}^{\prime}$ have the same tropes because of the way we identify the 15 nodes. Since each pair of tropes intersects at exactly 2 points, actually nodes, there has to be some pair of tropes that meet at only one node of the previously identified 15 nodes, in which case the second point of intersection is the last and sixteenth node. Thus, the two Kummer surfaces are actually equal.

This proposition can be restated as follows: in the projective space $\mathbb{P}^{3}$, six points in general position define a curve $C$ of genus two. The complete linear system of quadric surfaces through those 6 points defines a rational double cover of another $\mathbb{P}^{3}$ whose branch locus is the Kummer quartic surface of $C$.

As an immediate corollary of the proof, we obtain:

Corollary 4.4.5. The twisted cubic curve through the 6 theta characteristics of $\check{\mathbb{P}}_{-}^{3}$ is contracted under the dual map $\mathcal{D}$ to the point of tangency of $V_{0}$ with the Igusa-Segre quartic $\mathcal{I}_{4}$. 


\section{REFERENCES}

[Ati57] M. F. Atiyah, Vector bundles over an elliptic curve, Proc. London Math. Soc. (3) 7 (1957), 414-452.

[Bak22] H. Baker, Principles of geometry, Cambridge Univ. Press, Cambridge, 1922.

[Bal90] V. Balaji, Intermediate jacobians of some moduli spaces of vector bundles on curves, Amer. J. Math. 112 (1990), 611-630.

[Bar95] W. Barth, Quadratic equations for level-3 abelian surfaces, in "Abelian varieties" (Egloffstein, 1993), de Gruyter, Berlin, 1995, pp. 1-18.

[Bea03] A. Beauville, The Coble hypersurfaces, C. R. Acad. Sci. Paris, Ser. I 336 (2003), no. 3, 189-194.

[BNR89] A. Beauville, M. S. Narasimhan, and S. Ramanan, Spectral curves and the generalised theta divisor, J. reine angew. Math. 398 (1989), 169-179.

[Cob17] A. Coble, Point sets and allied Cremona groups III, Trans. Amer. Math. Soc. 18 (1917), 331-372.

[Cob61] _ Algebraic geometry and theta functions, American Mathematical Society Colloquium Publication, vol. X, Amer. Math. Soc., Providence, RI, 1961, Revised reprint of the 1929 original.

[DN89] J. M. Drezet and M. S. Narasimhan, Groupe de Picard des variétés de modules de fibrés semi-stables sur les courbes algébriques, Invent. Math. 97 (1989), 53-94.

[EL92] L. Ein and R. Lazarsfeld, Stability and restrictions of Picard bundles, with an application to the normal bundles of elliptic curves, in "Complex Projective Geometry", Cambridge University Press, 1992, pp. 149-156.

[FH79] W. Fulton and J. Hansen, A connectedness theorem for projective varities, with applications to intersections and singularities of mappings, Ann. of Math. 110 (1979), no. 1, 159-166.

[Gee82] G. van der Geer, On the geometry of a Siegel modular threefold, Math. Ann. 260 (1982), no. 3, 317350.

[GKZ94] I. M. Gelfand, M. M. Kapranov, and A. V. Zelevinski, Discriminants, resultants, and multidimensional detreminants, Mathematics: Theories \& Applications, Birkhauser Boston, Inc., Boston, MA, 1994.

[GLP83] L. Gruson, R. Lazarsfeld, and C. Peskine, On a theorem of Castelnuovo, and the equations defining space curves, Invent. Math. 72 (1983), no. 3, 491-506.

[Gro68] A. Grothendieck, Cohomologie locale des faisceaux cohérents et théorèmes de Lefschetz locaux et globaux ( $S$ GA 2), North-Holland Publishing Co., Amsterdam; Masson \& Cie, Editeur, Paris, 1968.

[HR04] J.-M. Hwang and S. Ramanan, Hecke curves and Hitchin discriminant, Ann. Sci. Ecole Norm. Sup. (4) 37 (2004), no. 5, 801-817.

[Hud90] R. W. H. T. Hudson, Kummer's quartic surface, Cambridge University Press, Cambridge, 1990, Revised reprint of the 1905 original.

[Koi76] S. Koizumi, Theta relations and projective normality of abelian varieties, Amer. J. Math. 98 (1976), 865-889.

[KP95] A. Kouvidakis and T. Pantev, The automorphism group of the moduli space of semi stable vector bundles, Math. Ann. 302 (1995), no. 2, 225-268.

[Las96] Y. Laszlo, Local structure of the moduli space of vector bundles over curves, Comment. Math. Helv. 71 (1996), no. 3, 373-401.

[LB92] H. Lange and C. Birkenhake, Complex abelian varieties, Springer-Verlag, 1992.

[MN68] D. Mumford and P. E. Newstead, Periods of a moduli space of bundles on curves, Amer. J. Math. 90 (1968), 1201-1208.

[New68] P. E. Newstead, Stable bundles of rank 2 and odd degree over a curve of genus 2, Topology 7 (1968), 205-215.

[Ngu05] Q. M. Nguyễn, Dualities and classical geometry of the moduli space of rank 3 vector bundle on a curve of genus 2, Ph.D dissertation, University of Michigan, 2005.

[NR69] M. S. Narasimhan and S. Ramanan, Moduli of vector bundles on a compact Riemann surface, Ann. of Math. 89 (1969), 14-51.

[NR75] Deformations of the moduli space of vector bundles on a curve, Ann. of Math. 101 (1975), 391-417.

[NR87] 2 20-linear system on abelian varieties, Vector bundles on algebraic varieties (Bombay 1984), Tata Inst. Fund. Res. Stud. Math., vol. 11, Tata Inst. Fund. Res., Bombay, 1987, pp. 415-427.

[NR03] Q. M. Nguyê̂n and S. Rams, On the geometry of the Coble-Dolgachev sextic, Le Matematiche (Catania) 58 (2003), no. 2, 257-275.

[OO05] A. Ortega Ortega, On the moduli space of rank 3 vector bundles on a genus 2 curve and the Coble cubic, J. Algebraic Geom. 14 (2005), no. 2, 327-356.

[Pau02] C. Pauly, Self-duality of Coble's quartic hypersurface and application, Michigan Math. J. 50 (2002), 551-574.

[Ses67] C. S. Seshadri, Space of unitary vector bundles on a compact Riemann surface, Ann. of Math. 85 (1967), 303-336. 
[Tu93] L. W. Tu, Semistable bundles over an elliptic curve, Adv. Math. 98 (1993), 1-26.

[Tyu74] A. N. Tyurin, The geometry of moduli of vector bundles, Russ. Math. Surveys 29 (1974), no. 6, 57-88.

[Wed50] T. Weddle, On the theorems in space analogous to those of Pascal and Brianchon in a plane.-part II, Camb. and Dub. Math. Jour. 5 (1850), 58-69.

3065 JACKSON ST, SAN FRANCISCO, CA 94115 (USA)

E-mail address: quang-minh.nguyen@sfuhs.org 